\title{
L'hégémonie politique et économique comme cadre de diffusion des techniques de construction au Moyen Age: la Corse entre Toscane et Ligurie du $\mathrm{xI}^{\mathrm{e}}$ au $\mathrm{XIV}^{\mathrm{e}} \mathrm{s}$.
}

\author{
DANIEL ISTRIA*
}

\begin{abstract}
Résumé
La Corse médiévale est une région où peu de réalisations majeures voient le jour dans le domaine de l'architecture. Elle n'en demeure pas moins, compte tenu de sa position et du contexte politique, d'un intérêt certain pour l'étude des échanges et des transferts de savoir-faire. La construction d'un important réseau d'édifices de culte à partir de la fin du xie siècle sous le contrôle de l'archevêque de Pise, permet l'introduction, puis la diffusion, de procédés techniques élaborés sur les grands chantiers continentaux, particulièrement toscans. C'est alors une véritable renaissance de l'architecture que connaît la Corse.
\end{abstract}

Mots clés: Diffusion, techniques de construction, Corse, Moyen Age

\begin{abstract}
Medieval Corsica is an area where few major architectural constructions have been developing. Nevertheless thanks to its geographical position as well as to the overwhelming political context, it does possess an undeniable importance if we focus on such issues as trade and the transfers of the know-how. Indeed the construction, from the $11^{\text {th }}$ under the control of the archbishop of Pisa, of a considerable network of building devoted to worship, leads to the introduction and consequently to the spread of technical processes engineered in the most renowned building sites on the mainland and more particularly in Tuscany. Corsica is thus experiencing a complete architectural rebirth.
\end{abstract}

Key words: Diffusion, building techniques, Corsica, Middle Ages

\footnotetext{
* Je tiens à exrpimer ma reconnaissance à J.A. Quiros Castillo pour m’avoir invité à participer à cette publication collective et à remercier François Bougard, Philippe Bernardi et Agnès Bonnet pour leurs très nombreux conseils.
}

En Corse, l'archéologie du bâti est une discipline extrêmement récente qui n’a réellement été mise en œuvre qu’à partir de la fin des années $1990^{1}$. Depuis 1967 les chercheurs insulaires disposent pourtant d'un copieux inventaire, même si non exhaustif, des édifices de culte médiévaux qui a servi de base à une étude strictement stylistique à l'origine d'une première typo-chronologie de l'architecture religieuse, rapidement étendue au domaine castral (MORACCHINI-MAZEL 1967 et 1984). Les datations proposées dans cette étude ont été remises en question depuis la fin des années 1970, en raison de leur mode même d'élaboration (PeRGOLA 1979, 1980a et b). Mais, au-delà du questionnement lié à la chronologie du monument, qui reste un élément fondamental de la recherche, les études s'orientent aujourd'hui vers l'analyse des matériaux et des techniques de construction, en tentant de les replacer dans leur contexte historique, social et économique ${ }^{2}$.

L'évolution des savoir-faire et des modes de mise en œuvre est avant toute chose le fruit des échanges et des relations entre les hommes. En Corse, comme dans tout milieu insulaire, les contacts avec l'extérieur ont été le moteur de cette évolution. La mer peut aussi bien être perçue et utilisée comme une large ouverture, en l'occurrence sur le bassin occidental de la Méditerranée et plus particulièrement sur l'aire tyrrhénienne, que comme une barrière, un frein, aux déplacements des hommes et des idées. Ainsi, après plusieurs siècles de difficultés liées à la présence sarrasine le long des côtes, sinon à l'intérieur même des terres (Pergola 1999: 24), la Corse entre, avec le Moyen Age, dans une période féconde durant laquelle des relations politiques et économiques, solides et durables, vont se mettre en place avec les cités de Pise et de Gênes.

En 1077 le pape Grégoire VII tente de réaffirmer l'autorité pontificale sur l'île en confiant sa gestion, par délégation de pouvoirs, à l'évêque de Pise. Les Pisans s'appliquent alors à mettre en place ou à renforcer la puissance des seigneurs insulaires tout en cherchant à les garder sous leur tutelle. Mais, l'intervention génoise conduit très tôt à un revirement de situation avec, en 1133, le partage des évêchés entre les deux républiques. A Pise échoit le sud de l'île, alors que Gênes hérite des diocèses de Mariana, Nebbio et Accia situés dans la moitié nord. Si Pise conserve des alliances avec de puissantes familles seigneuriales du nord de l'île à l'exception du Cap Corse contrôlé par des lignages

\footnotetext{
1 Pour une approche générale du concept d'archéologie du bâti, c'est-à-dire «l'étude architecturale des élévations qui ne se limite pas à des considérations purement stylistiques» et des grandes orientations de cette discipline sur le territoire français voir en premier lieu: ESQUIEU 1997.

2 Voir en particulier l'étude réalisée par l'équipe de l'université de Florence, dirigée par Guido Vannini dans le cadre de Projet Collectif de Recherche «Mariana et la basse vallée du Golo».
} 
ligures installés là depuis le XIIe siècle, Gênes développe des activités commerciales dans le sud grâce à la fondation de la colonie de Bonifacio, puis dans le nord-ouest, après s'être emparée de la place forte de Calvi. Jusqu’à la fin du XIIIe siècle il est bien difficile de dire qui possède une réelle autorité sur l'île. L'hégémonie de la cité ligure ne se fait vraiment ressentir qu'après la victoire de la Meloria en 1284. Cinq ans plus tard, en 1289, Lucchetto Doria est envoyé avec le titre de vicaire général de la commune et avec pour mission de soumettre les seigneurs insulaires. Malgré le succès de l'expédition, la partie n'est pas pour autant gagnée. La situation se complique en effet lorsque, le 17 février 1297, le pape Boniface VIII investit Jacques d'Aragon des royaumes de Corse et de Sardaigne. Cette situation confuse et la multiplicité des forces en présence, engendrent un climat d'anarchie qui régnera durant toute la première moitié du XIVe siècle malgré la présence dans l'île de gouverneurs nommés par Gênes. En 1357, profitant d'une période d'instabilité et de crise, la cité ligure fomente une révolte avec pour objectif le renversement des puissantes familles seigneuriales insulaires. Ce mouvement populaire aboutit rapidement à la destruction des châteaux, symboles du pouvoir sur les hommes et à la donation officielle de l'île à Gênes en 1358. Cette série d'événements marque un moment de crise qui conduit à une déstabilisation du pouvoir et à un conflit permanent. Il engendre de manière incontestable des bouleversements économiques mais surtout politiques qui aboutissent dès la seconde moitié du XIVe siècle à l'émergence de nouveaux rapports sociaux.

Dans le cadre de ces relations entre la Corse et l'ensemble toscano-ligure au Moyen Age, émerge la problématique fondamentale de la transmission des savoirs techniques et plus exactement de la dialectique tradition/innovation qui nous conduit à une réflexion sur le rôle du rapport de dépendance de l'île vis-à-vis des deux puissances maritimes, vecteurs de la diffusion de ces savoirs. Il importe, alors, d'évaluer la part des acquisitions techniques dans l'île du XIe au XIVe siècle, de comprendre leur mode de diffusion, mais aussi la capacité de la société insulaire à intégrer les nouveautés et à les perpétuer.

Pour tenter d'apporter quelques éléments de réponse à ces interrogations, nous nous intéresserons ici aux problèmes des matériaux, de leur choix, de leur préparation et de leur mise en ouvre, à partir de l'observation de plusieurs monuments civils, militaires ou religieux, construits dans toute l'île entre le XIe et le XIVe siècle 3 .

${ }^{3}$ Cette étude est tirée d'une thèse soutenue à l'université d'Aix-Marseille I en 2000: Istria D., Châteaux et habitats fortifiés dans le nord de la Corse (1077-1358). Pour une approche plus approfondie de la question nous renvoyons à l'étude en cours sous la direction de Guido Vannini (cité note 2). Voir aussi ISTRIA 2005.

\section{INTRODUCTION ET DIFFUSION DE L'ARCHITECTURE ROMANE ET DES NOUVELLES TECHNIQUES DE CONSTRUCTION (FIN XIE-XIIE SIĖCLE)}

L'architecture insulaire du haut Moyen Age est encore très peu documentée $e^{4}$ Toutefois, l'exemple de la basilique de Mariana peut suffire à prendre conscience de l'importance des changements introduits dans l'île à partir de la seconde moitié du XIe siècle.

La colonie romaine de Mariana fut fondée vers 90 av. J.-C. Elle devient à l'époque impériale la principale cité de l'île et le resta, sans doute, durant tout le Moyen Age en raison de son statut de ville épiscopale 5 .

Une première basilique à trois nefs, terminée vers l'est par une abside semi-circulaire fut érigée ici, sur un quartier d'habitation antique, vers la seconde moitié du ve siècle (Pergola et Di Renzo 2001). Cet édifice fut remanié à plusieurs reprises dans le courant du haut Moyen Age. Les derniers travaux ont bouleversé totalement l'aspect du sanctuaire, désormais restreint à la seule nef centrale, limitée à l'ouest par un nouveau mur beaucoup plus près du chœur que la précédente façade. L'espace qui subsistait entre les piles de briques qui ont remplacé les vielles colonnes en granite de la nef est alors obturé. Les nouvelles maçonneries sont caractérisées par le remploi systématique de matériaux de construction: briques, tegulae mais aussi fragments de marbre décorés d'époque impériale et du VIIIe siècle (Pergola et Di Renzo 2001). Le liant utilisé est de l'argile et non plus du mortier de chaux, comme c'était le cas dans les maçonneries primitives (DUVAL 1995: 349). Il s'agit donc d'une construction de facture grossière et maladroite, réalisée sans doute à peu de frais.

La décision de Grégoire VII de réaffirmer la souveraineté pontificale sur la Corse est à l'origine de bouleversements

\footnotetext{
${ }^{4}$ Les datations très hautes (du Ve au Xe siècle) proposées par G. Moracchini-Mazel (1967) pour toute une série d'églises insulaires sont à rejeter puisqu'elles reposent essentiellement sur des textes antidatés (principalement ceux du cartulaire de l'abbaye de Montechristo).

${ }^{5}$ Le siège épiscopal de Mariana apparaît au travers de la documentation écrite comme un centre privilégié, tant sur le plan religieux que politique et monumental. Ainsi, en 1116, s'y déroule un concile provincial en présence du cardinal Rolando, légat pontifical, et du marquis de Corse Ugo (Archives départementales de Corse, IHI, 6, 22 décembre 1116). En 1118, un nouveau concile est tenu à Mariana sous la présidence de Pierre, cardinal de S. Susanna, légat pontifical. L'acte est rédigé à Mariana en présence de Petrus, archevêque de Pise et des évêques de Mariana, Aleria et Nebbio (Archives départementales de Corse, IHI, 7, 1119). C'est aussi la seule cathédrale (et église) corse à être consacrée solennellement par l'archevêque de Pise, Petrus et le cardinal Petrus de S. Susanna, légat pontifical, en présence des chanoines de Pise, d'un consul et juge pisan, de citoyens pisans, du peuple et des ecclésiastiques de Corse qui jurèrent fidélité au Pape (Muratori 1725, tom. VI, col. 105). Sur le plan politique, on soulignera simplement un évènement important à valeur fortement symbolique: l'élection du comte de Corse Giudice de Cinarca au milieu du XIIIe siècle.
} 
Fig. 1. Lucciana, cathédrale de Mariana, vue de l'église et de la tour

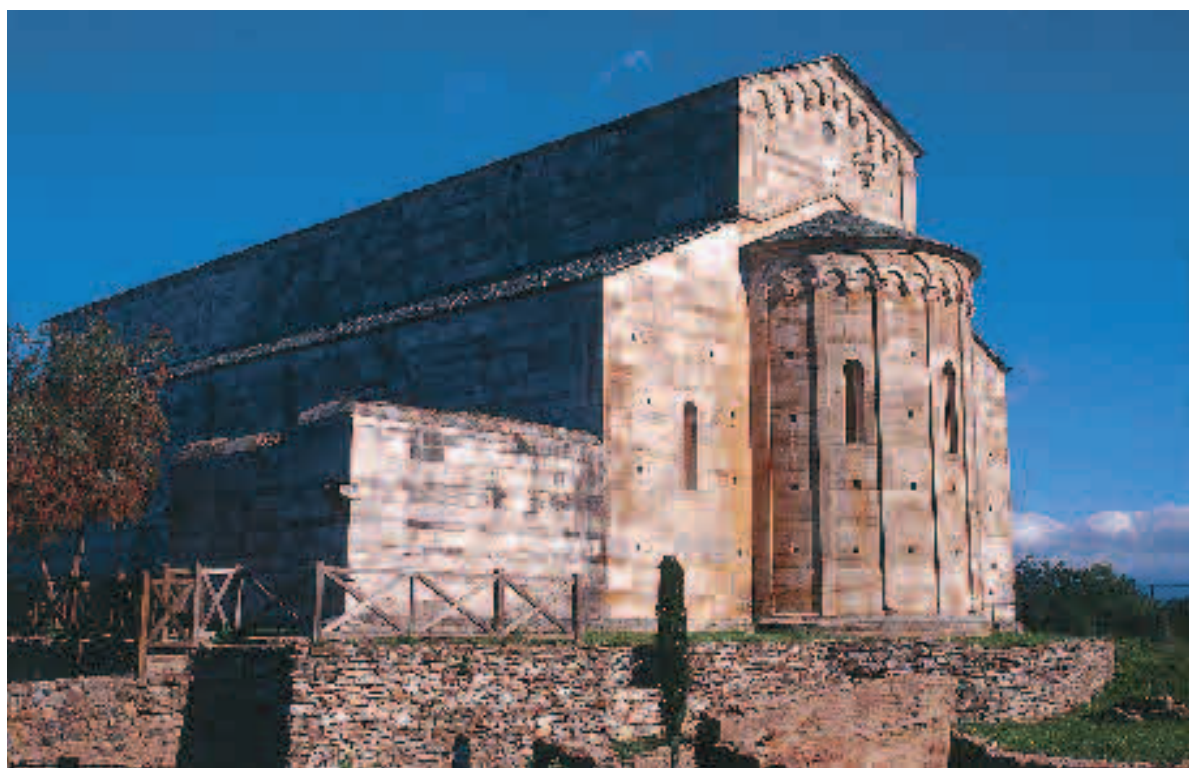

politiques importants mais il faut attendre 1092 pour que l'évêque de Pise soit promu au rang d'archevêque avec pouvoirs métropolitains. Cet évènement entraîne rapidement un spectaculaire renouveau de l'Eglise corse (ISTRIA et DI RENZO 2001). Les anciens évêchés sont réactivés et un important réseau de pievi est mis en place dans un contexte fondamentalement rural. A la tête de chacune de ces circonscriptions s'élève désormais une église, le plus souvent fondée ex nibilo, rapidement relayée par des édifices de culte secondaires dont on attribue la construction aussi bien aux évêques qu’à de puissants lignages laïcs. Ce vaste mouvement général de construction —on estime aujourd'hui à environ 800 le nombre d'églises romanes dans l'île— a permis l'introduction de modes de bâtir totalement nouveaux.

La cathédrale de Mariana est reconstruite à quelques mètres au nord de la précédente (Fig. 1). La nouvelle église, dédiée à Santa Maria Assunta, est édifiée sur un plan à trois vaisseaux $(35 \times 14 \mathrm{~m})$ charpentés, séparés par des piles de section quadrangulaire recevant les retombées des arcs plein cintre. Elle fut consacrée en 1119 (MURATORI 1725, col. 105). Elle s'oppose à la précédente église par la qualité de la construction des murs à double parement dont les dalles de schiste cipolin, provenant du Cap Corse (environ $20 \mathrm{~km}$ ), ont fait l'objet d'un traitement de surface optimal à la broche ou, dans de rare cas, à la gradine ${ }^{6}$. Les ciselures

${ }^{6}$ Des outils de tailleur de pierre ont été retrouvés lors des fouilles «à proximité de la cathédrale et dans d'épaisses couches de petits éclats de pierre de Brando»: MORACCHINI 1967: 84. L'auteur signale «un ciseau de marbrier à six dents». Des traces de cet outil sont visibles, en particulier, sur les archivoltes des fenêtres, sur le tympan de la porte sud-est ainsi que sur plusieurs claveaux. Quelques blocs de parement de la cathédrale et de l'é- périmétrales étant réalisées au ciseau droit. Ces pierres servent de revêtement à une fourrure constituée de galets ou de moellons noyés dans du mortier de chaux résistant (MoraCCHNi-MAZEL 1967: 84). Elles ont été posées de manière à faire alterner des assises hautes et minces selon un rythme irrégulier créant un effet de lignes horizontales, accentué par l'utilisation de pierres de tonalité légèrement différente. Les lésènes sont constituées de blocs pourvus de retours, ou d'ailettes, disposés verticalement. Ce procédé, destiné à renforcer la structure des murs, qui ne se retrouve que sur quelques rares édifices de la région de Pise et en Sardaigne, témoigne du haut niveau de connaissance des bâtisseurs ${ }^{7}$. Les décors sculptés sont peu nombreux, très sobres et de factures diversifiées. On remarquera en particulier les trois blocs insérés dans la face sud et sculptés de motifs géométriques «a intarsio» (Fig. 2) absolument identiques à ceux qui sont visibles à Pise, sur la façade de la cathédrale et sur le portail de l'église San Sepolcro, mais aussi en Sardaigne, sur le bras sud de l'édifice Santa Maria di Castello à Cagliari (MORACCHNi-MAZEL 1967: 87-94).

Par ses proportions, le rythme des façades mais aussi la mise en œuvre de certains procédés techniques, la cathédrale de Mariana doit être incontestablement rapprochée de l'église Santa Maria del Regno d'Ardara, en Sardaigne, dont la consécration eut lieu en 1107 (CORONEO 1993).

glise San Parteo — dont la nef est probablement construite par l'équipe de la cathédrale- semblent également porter des traces de bretture mais la nature de la roche — du schiste cipolin_ ne permet pas d'être absolument certain de cette identification.

7 Ce procédé a également été mis en œuvre lors de la construction de l'abside de la cathédrale de Nebbio. 


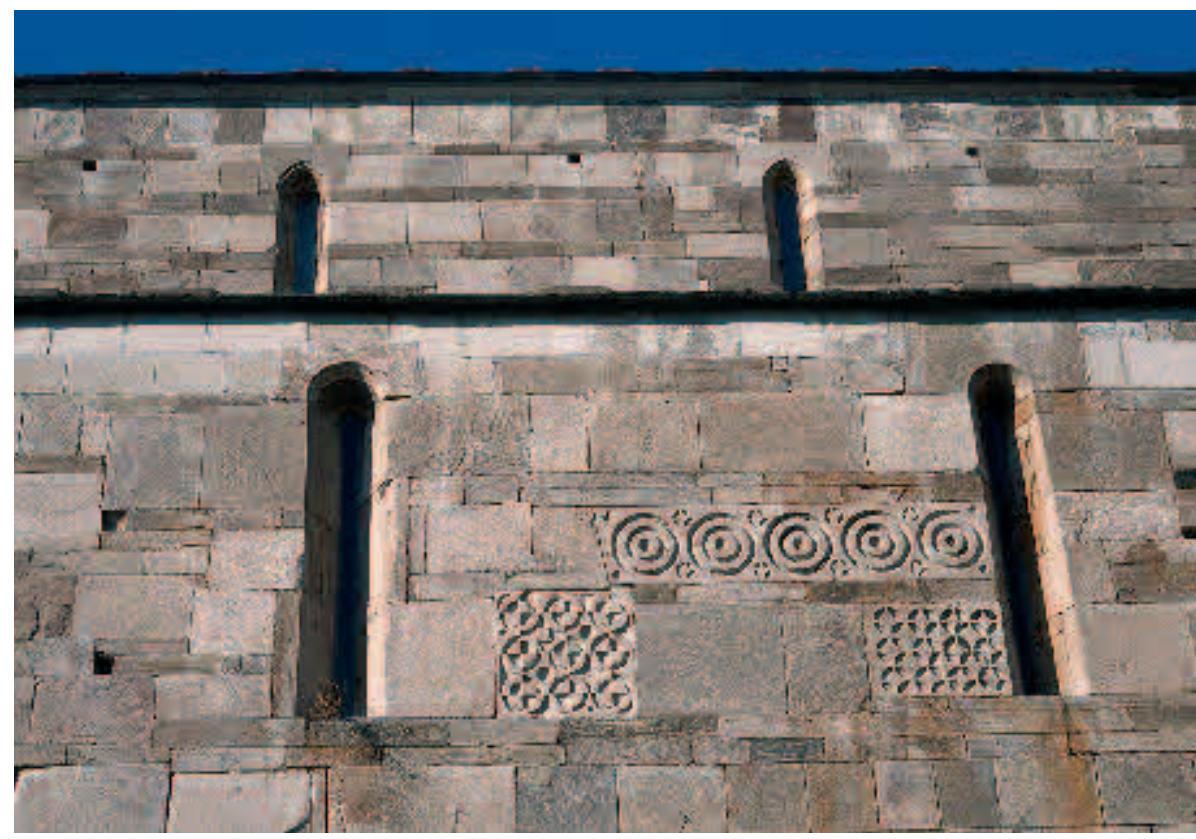

Fig. 2. Lucciana, cathédrale de Mariana, détail du mur sud de la cathédrale
Ces deux monuments forment un groupe très cohérent, caractérisé à la fois par un style emprunté à l'église San Sisto de Pise, construite durant le dernier quart du XIe siècle (REDI 1991: 359), et par la mise en œuvre systématique de techniques de taille de la pierre qui semblent élaborées sur le chantier de la cathédrale de Pise (CORONEO 2004).

\section{DIFFUSION ET DIVERSIFICATION DES TECHNIQUES DE TAILLE DE LA PIERRE}

Avec la construction de ce monument au tout début du XIIe siècle, il est possible de suivre l'introduction —ou plus exactement la réintroduction- dans l'île de deux éléments importants: la technique de taille de la pierre (et donc de nouveaux outils), ainsi que le mortier de chaux. Tous deux se retrouvent désormais utilisés de manière quasi systématique sur les chantiers d'édifices de culte durant tout le XIIe siècle ${ }^{8}$. Pourtant, ces techniques ne vont pas se figer.

8 G. Bianchi présente un cas fort intéressant de la diffusion de la pierre de taille en Toscane entre la fin du XIe et le début du XIIe siècle — donc au même moment qu'en Corse- à partir de l'exemple du site de rocca San Silvestro (BIANCHI 1995: 377). En Corse, nous ne connaissons, à l'heure actuelle, qu'un seul édifice de culte pouvant être attribué sûrement au XIIIe siècle. Il s'agit de la chapelle San Michele située dans la pieve de Vallerustie (commune de San Lorenzo). En 1875, un reliquaire fut découvert dans l'autel de cet édifice. Il portait le sceau de l'évêque d'Aleria Attolinus qui pourrait correspondre à l'Attolinus mentionné dans un document de 1256: Moracchini-Mazel 1967: 321. Selon Edouard Bouyé, il ne fait aucun doute que ce sceau puisse être du milieu du XIIIe siècle (information orale). Cette situation s'oppose radicalement à celle de la Sardaigne où de nombreuses églises sont édifiées au XIIIe siècle mais dans un style largement influencé par le gothique.
A partir du second quart du XIIe siècle environ, on assiste dans le diocèse de Nebbio à une véritable fleuraison d'édifices de culte. Il s'agit alors d'un moment très faste pour cet évêché marqué par l'implantation de l'abbaye Toscane de San Gorgonio de l'île de la Gorgona et par un développement des activités économiques sous l'influence de quelques évêques dynamiques (ISTRIA 2005). Deux séries d'églises semblent construites simultanément par autant d'équipes qui ont élaboré un style différent bien que présentant un certain nombre de points communs.

La cathédrale de Nebbio est contemporaine de celle de Mariana (Fig. 3). Contrairement à cette dernière, elle fut bâtie sur le modèle "buschetiano», parfaitement illustré par les églises pisanes de San Pietro in Vincoli et San Frediano par exemple. Cette cathédrale est probablement à l'origine de la série d'édifices liés à San Michele de Murato (Fig. 4) ${ }^{9}$. La parenté des deux, sans être évidente de prime abord, paraît très probable en raison de la mise en œuvre de certains partis architecturaux, comme l'animation de la façade occidentale par de grandes arcatures aveugles et la composition des chapiteaux. Les blocs de parement sont ici d'une qualité remarquable. Ils sont assemblés en assises très régulières. Les deux types de pierre utilisés proviennent de carrières relativement éloignées: la serpentine a été prélevée dans la vallée du Bevinco, à envi-

9 L'église San Michele de Murato est mentionnée pour la première fois en 1137 (Archives départementales de Corse, Bastia: 1H1, 13, 31 mars 1137). 
ron 5 ou $6 \mathrm{~km}$ de la chapelle, et le calcaire provient de Saint-Florent (environ $15 \mathrm{~km}$ ). Les motifs choisis pour décorer les modillons, sur lesquels retombent les petits arcs aveugles, sont répétitifs, d'une facture sèche et, assez souvent même, naïve. Ce décor est complété par des sculptures en très fort relief, voire en ronde bosse et en bas relief sur les corniches, les tympans, les linteaux ou les appuis des fenêtres. L'animation murale, constituée de bandes arcaturées couronnant les murs et surtout de grandes arcatures aveugles en façade, renvoie aux modèles pisans simplifiés à l'extrême. On les retrouve en Sardaigne avec quelques variantes, peut-être plus proches des modèles originaux (San Nicolo d'Ottana, Santa Maria de Bonocardo...).

On doit rapprocher de cette série quelques édifices de la région, comme Santa Trinita d'Aregno et surtout San Quilico de Cambia.

Le second groupe d'églises, quantitativement très important dans le nord de l'île, possède les mêmes caractéristiques techniques que le précédent. Les seules différences se situent au niveau des décors. Ils se caractérisent ici par la présence sous les bandes arcaturées de sculptures en fort relief, au trait sec et anguleux. Les linteaux et les consoles des portes principales, sculptés de motifs antiquisants très schématisés - dérivés des feuilles d'acanthe, d'oves, de rinceaux... - s'apparentent fortement à ce que l'on peut observer sur les édifices toscans comme San Giorgio de Brancoli, mais on soulignera aussi l'étonnante ressemblance avec les décors et la forme des ouvertures des édifices également toscans un peu plus anciens comme la pieve de Vico-

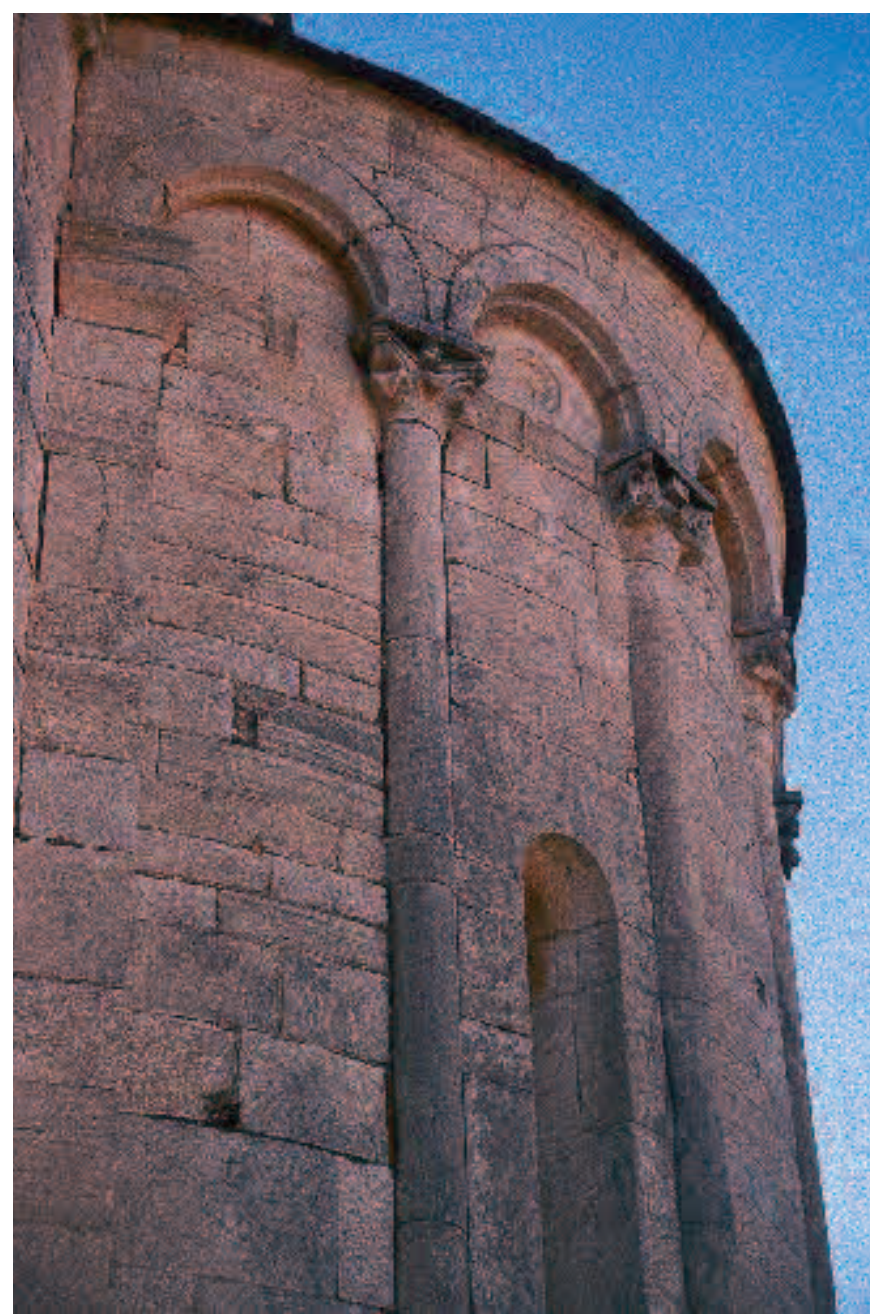

Fig. 3. Saint-Florent, cathédrale de Nebbio, détail de l'abside. On remarque, à mi-hauteur des colonnes, les blocs avec retours destinés à rigidifier la structure

Fig. 4. Murato, église San Michele, détail du mur nord. On notera les traces d'outils visibles sur les pierres

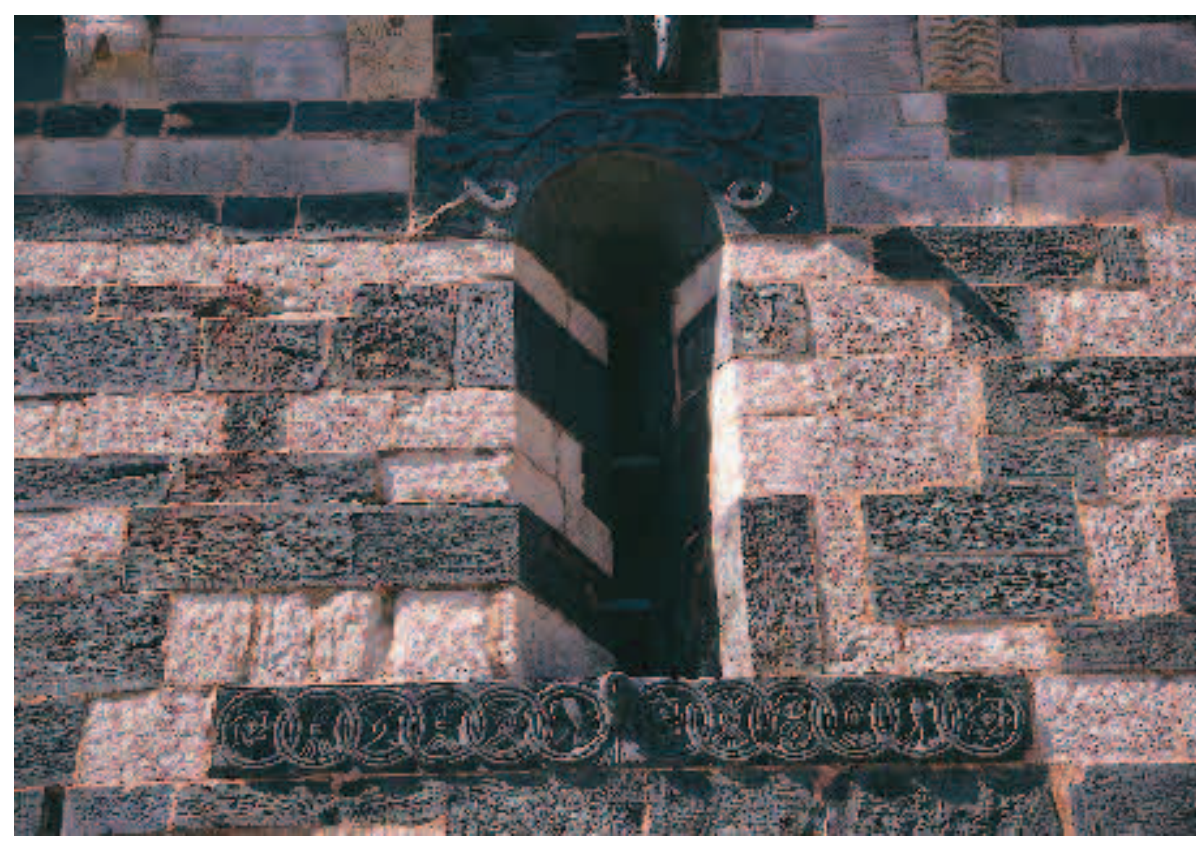


pisano ou encore l'église San Iacopo de Zambra (REDI 1984, vol. I: 148) ${ }^{10}$.

Si on peut être quasiment certain que les pievi Santa Maria de Canari et Santo Pietro di Tenda ${ }^{11}$, toutes deux situées dans le diocèse de Nebbio, sont de la main d'une même équipe de bâtisseurs, il est difficile de dire si les édifices plus modestes que sont San Pietro de Giuncaggio, San Sarsorio de Bastia, Sant'Andrea de Biguglia et Santa Margarita de Sorio, sont l'œuvre d'une équipe différente, moins habile, peut-être issue de la première, ou s'il s'agit des mêmes maçons qui ont travaillé avec des moyens moins importants.

Sur le plan strictement technique, ces équipes de bâtisseurs introduisent l'usage de quatre outils peu ou pas utilisés auparavant: la hache et le trépan, dont l'usage reste très limité ${ }^{12}$, et surtout la gradine et la bretture ${ }^{13}$, toujours associées à des bordures taillées au ciseau droit (Fig. 4) ${ }^{14}$. La large diffusion de la lame dentée pourrait n'être que le résultat d'une évolution opérée sur le chantier de la cathédrale de Pise à la fin de la campagne de construction dirigée par Buschetto (FABIANI et al. 1997; PARENTI et al. 1997). Cette innovation fut adoptée par son successeur Rainaldo, entre 1115 et 1130 environ (REDI 1991: 355), et par quelques équipes itinérantes qui sont restées plus ou moins fidèles au schéma architectural imaginé par Buschetto ${ }^{15}$.

D'autre part, on constate que l'échafaudage indépendant à deux rangs de perches se substitut à l'échafaudage encastré utilisé par exemple à Mariana. Ce nouveau choix,

\footnotetext{
10 Ces églises ont été datées des années 1120.

${ }^{11}$ Ces deux églises sont mentionnées pour la première fois respectivement en 1124 (Archives départementales de Corse, Bastia 1H1, 8, 6 janvier 1124) et en 1137 (Archives départementales de Corse, Bastia 1H1, 13, 31 mars 1137).

${ }^{12}$ Les traces de ces deux outils sont visibles sur les églises San Cesario de Rapale et Sant'Agostino de Bigorno. On trouve aussi des traces d'une taille en chevrons à la hache sur les parements de la pieve San Giovanni de Cursa. Pour une description de ces édifices construits probablement à partir du second tiers du XIIe siècle voir: MORACCHINI-MAZEL 1967: 150-151 et 337-338; IsTRIA et DI RENZO 2001.

13 Cette outil a laissé des traces sur de nombreux monuments et particulièrement sur les parements de San Michele de Murato. Ici, il s'agit de traces linéaires de 2 à $5 \mathrm{~cm}$ de long environ, à l'intérieur desquelles on peut remarquer des stries parallèles correspondant aux marques des dents. Dans deux cas, on a pu calculer les dimensions des taillants et des dents de gradines:

- Largeur du taillant environ 2,4 cm, 4 dents de $5 \mathrm{~mm}$ environ, espacées de $1 \mathrm{~mm}$.

- Largeur du taillant environ $2 \mathrm{~cm}, 5$ dents de $3 \mathrm{~mm}$ espacées de $1,5 \mathrm{~mm}$. Dans d'autres cas, on a pu mesurer des dents de 2 à $3 \mathrm{~mm}$ de largeur.

${ }_{14}$ Ces bordures, d'une largeur proche de $2 \mathrm{~cm}$, sont en effet marquées par des traces de ciselures de dégagement obliques et pratiquement parallèles.

15 Il serait utile de fixer précisément la date d'introduction de la gradine et de la bretture ainsi que la chronologie et la géographie de leur diffusion. Pise semble, à l'heure actuelle, être un centre précurseur.
}

qui respecte les parements de l'édifice, semble découlé d'une volonté esthétique puisque les trous de boulins, auparavant laissés apparents dans les murs, sont désormais supprimés (BAUD et al. 1996: 27). Malgré les problèmes de sécurité qu'il pose, il permet aussi d'économiser la découpe des blocs pour l'insertion des poutres horizontales et une récupération plus commode du bois d'œuvre ${ }^{16}$.

L'importation et le développement de ces techniques, tout comme la diffusion des styles romans pisans, témoignent incontestablement de la présence d'équipes de bâtisseurs itinérants formées en Toscane, voire à Pise même. Les nombreuses similitudes entre l'architecture religieuse Sarde et Corse permettent de penser que beaucoup de ces maçons ont, par ailleurs, travaillé dans les deux îles.

\section{LA PIERRE DE TAILLE DANS L'ARCHITECTURE CASTRALE}

La diffusion de ces nouvelles techniques se fait rapidement et à peu près uniformément sur l'ensemble de l'île. Néanmoins, leur emploi n'est pas systématique; sur certains chantiers, peu nombreux, l'utilisation de la pierre de taille reste très marginale. C'est le cas à Santa Maria de Rescamone et à San Giovanni de Venaco, par exemple (IsTRIA et Di RENZO 2001). Mais, c'est surtout dans l'architecture castrale que ce phénomène se manifeste de la manière la plus évidente. Seulement dix tours — sur plus de 120 recensées dans le nord de la Corse- sont bâtis en pierre de taille. Le travail est en général d'une qualité bien plus médiocre que celui réalisé pour l'architecture religieuse alors que les matériaux sont prélevés sur place, à l'exception de quelques très rares blocs de travertin intégrés dans les maçonneries. On remarque quelques variantes liées essentiellement au degré de finition des surfaces visibles, toujours travaillées à la broche et au ciseau droit. Néanmoins, on retrouve de nombreuses similitudes sur les deux types d'édifices (militaires et religieux), laissant ainsi transparaitre le travail de mêmes équipes. L'exemple le plus significatif est celui de Mariana. Une tour fut édifiée contre le mur latéral sud de la cathédrale probablement très peu de temps après sa consécration (Fig. 1) ${ }^{17}$. Les techniques et les matériaux utilisés sont strictement identiques. On remarque sur la face sud de cette petite construction de plan carré dont il ne reste plus aujourd'hui que le premier niveau d'environ $4 \mathrm{~m}$ de hauteur, la présence de trois consoles situées à plus de $3 \mathrm{~m}$ au-dessus

\footnotetext{
16 Les églises Sardes auxquelles nous avons renvoyé dans les lignes précédentes, sont marquées par une même évolution technique.

17 Il s'agit bien d'une tour défensive et non d'un clocher comme le prouve ses dimensions, l'absence de mur nord - les murs est et ouest s'appuient simplement sur l'église ce qui poserait des problèmes de stabilité s'il s'agissait d'un clocher — l'absence d'ouvertures — la porte se trouvait à au moins $3 \mathrm{~m}$ de hauteur- et l'absence de trous de boulin.
} 
du sol actuel. Deux sont situées près des angles et une, à peine plus large, au centre de la façade. Selon toute vraisemblance, elles constituaient les supports d'une terrasse en bois, située en hauteur. Un système absolument identique se retrouve sur de nombreuses façades pisanes des XIIe, XIIIe et XIVe siècles. Ainsi, pour la casa-torre Mazzarosa ${ }^{18}$ attribuée au XIIe siècle, et la domus di Mosca da San Gimigna$\mathrm{no}^{19}$ construite par un architecte florentin à partir de 1302, F. Redi restitue des terrasses fermées et couvertes. La console servant alors de support à un jambage oblique soutenant le plancher (REDI 1991: 180, tavola 20 et 188, tavola 23). Mais les exemples les plus proches se retrouvent dans un contexte rural, à quelques kilomètres de Pise, dans le village de Vicopisano. Plusieurs tours, dont en particulier la tour de l'horloge, y sont conservées et présentent de nombreuses analogies avec notre édifice de Mariana, tant en ce qui concerne les techniques de construction mises en œuvre que les dimensions ${ }^{20}$.

Un autre édifice du nord de la Corse, peut-être plus ancien que le précédent (ISTRIA 2000), présente de nombreuses analogies avec l'architecture péninsulaire. Il s'agit du donjon de château de San Colombano de Giussani. Bien que les parements de cette construction aient été arrachés au siècle dernier, on peut encore observer à la base des murs des blocs de calcaire liés à la chaux, parfaitement taillés à la broche et au ciseau droit. Outre cet appareillage de qualité, on notera ici la forme circulaire du donjon - il n'en existe que deux dans l'île- et la présence très probable d'un large fossée ${ }^{1}$. Le modèle de cette fortification est très certainement à rechercher en Lunigiana et plus particulièrement dans le Val di Magro (GALLO 1995, GALlo et MARSELLI 1989) d'où sont, justement, originaires les promoteurs du château.

Les éléments qui caractérisent ces deux fortifications sont révélateurs de l'importance et de la qualité des chantiers, en tout point comparables à ceux des grands édifices religieux. En revanche, l'analyse des vestiges des donjons de Stella, Montemagna, Longhetta, Serrevalle ou encore Rostino II, donne l'image d'une architecture moins raffinée. Si la pierre de taille est bien présente, elle est associée à des moellons bruts ou à peine retouchés au marteau têtu. Malgré l'utilisation de petites pierres de calages, les assises restent

\footnotetext{
18 Via Sta Maria n. ${ }^{\circ} 47-49$.

${ }^{19}$ Lungarno Gambacorti n. ${ }^{\circ}$ 2-3.

20 Il n'existe, à notre connaissance, qu'une seule étude archéologique du bâti médiéval de Vicopisano. Il présente l'intérêt de proposer un classement typologique précis mais les datations proposées restent, selon nous, à démontrer: ReDI et FANUCCI Lovitch 1998; ReDI 1997.

${ }^{21}$ Ce fossé, invisible actuellement en surface, a été repéré grâce à l'étude des photographies aériennes de l'IGN prises à haute altitude.
}

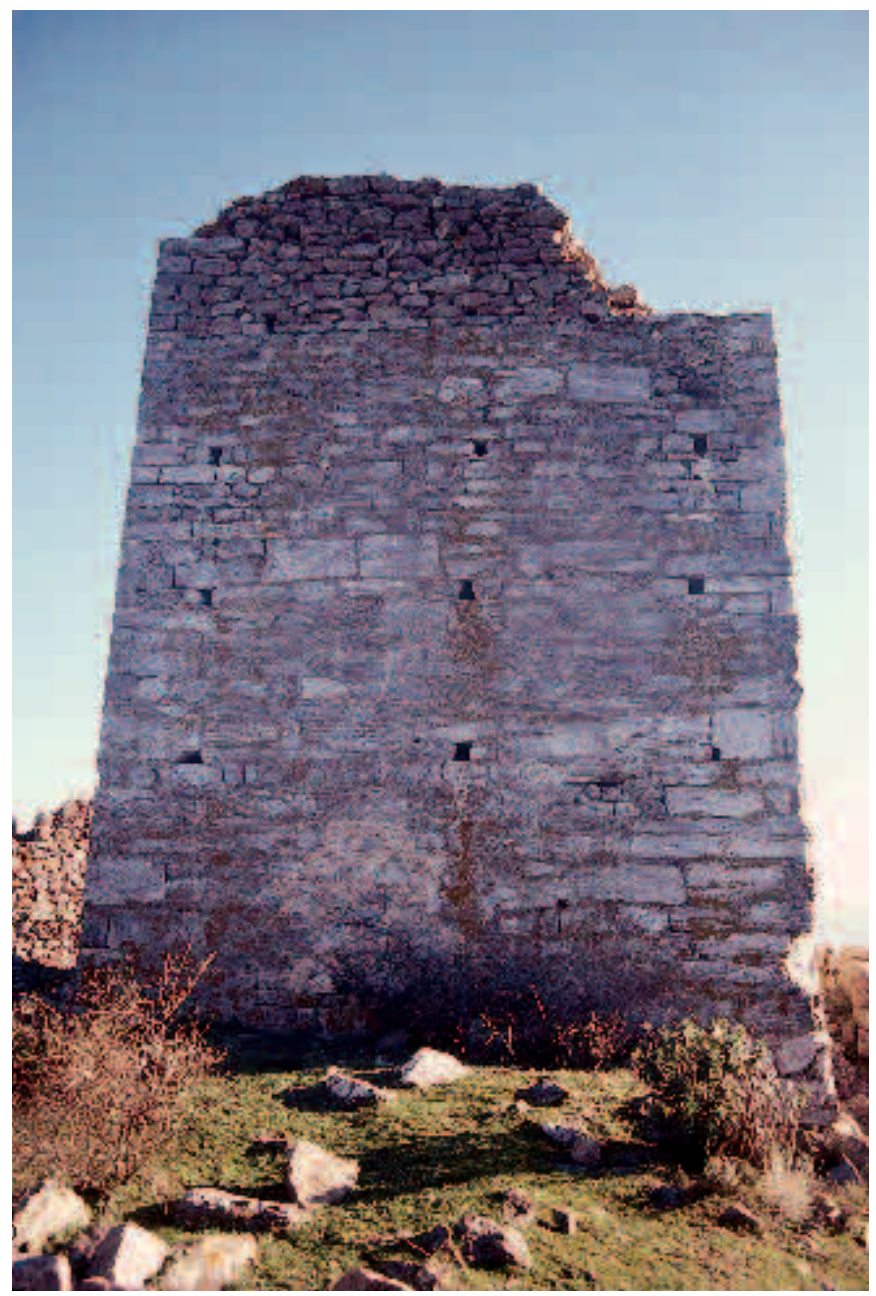

Fig. 5. Piedigriggio, château de Serravalle. Vue de la face sud du donjon

rarement horizontales et de hauteurs inégales. Dans tous les cas il semble que l'homogénéité et la régularité de l'appareillage soient étroitement liées aux possibilités offertes par les bancs géologiques du site à construire (Fig. 5).

Dans ce type d'édifices, les trous de boulins destinés à maintenir les échafaudages en bascule ou, le plus souvent, mixtes (BAUD et al. 1996: 27), sont maçonnés sans beaucoup de soin. L'exemple du donjon de Serravalle montre que les contraintes exercées par l'utilisation de perches - trois par face- ont obligé les maçons à adopter diverses solutions afin d'éviter de façonner un trou à l'intérieur d'un bloc, ce qui, par ailleurs, n’a pas toujours été possible. En effet, l'implantation des entrées étant déterminée par le montage de l'échafaudage, à deux reprises il a fallu tailler très grossièrement une échancrure pour $\mathrm{y}$ insérer la poutre. Du choix de ce système découlent des trous de boulins de formes et de dimensions très variées. Ils peuvent être carrés, rectangulaires - hauts et étroits ou larges et bas $(12 \times 15 \mathrm{~cm}$; $10 \times 24 \mathrm{~cm} ; 10 \times 21 \mathrm{~cm})$ - voire même trapézoïdaux. 
Quant aux trous façonnés ils sont étrangement de forme irrégulière, proche du quart de rond, ce qui témoigne probablement de la maladresse des tailleurs de pierre.

\section{LE DONJON II DE ROSTINO: COMPROMIS ENTRE TRADITION ET INNOVATION OU IMPORTATION D'UN MODĖLE PISAN?}

Le donjon II de Rostino présente des caractéristiques très particulières (Fig. 6). Il est installé, vers 1140-50 (IsTRIA 2000: 240-243 et ISTRIA 2005) à $642 \mathrm{~m}$ d'altitude sur un petit dôme rocheux qui couronne une butte d'éperon. Il s'agit d'une petite construction de plan trapézoïdal (dimensions intérieures $2 \times 2,5 \mathrm{~m}$ ) constituée d'un premier niveau conservé sur une hauteur de $1,80 \mathrm{~m}$ en moyenne, dont les murs de 1,40 à $1,50 \mathrm{~m}$ d'épaisseur sont construits en pierres liées à la chaux. Deux phases de construction ont été individualisées. Les murs nord-ouest et sud-ouest possèdent un parement extérieur soigné, constitué de blocs de schiste et plus rarement de travertin taillés à la broche et au ciseau droit, ainsi que de moellons plus ou moins réguliers prélevés dans les bancs géologiques présents sur le site même. De petits fragments de lauzes sont utilisés comme calages. Immédiatement après la mise en place de ces deux murs, de nouvelles techniques de construction sont adoptées. Les deux nouveaux murs sont alors bâtis en moellons non retouchés alors que les parois intérieures sont particulièrement irrégulières, tant sur le plan horizontal que vertical. Elles ne sont constituées que de moellons informes mal scellés par le mortier de chaux. Dès la pose des pre- mières assises, l'intérieur de la tour a été comblé par des déchets ménagers (cendre, charbons, matières organiques en décomposition) et des pierres, de manière à constituer une sorte de support, voire de coffrage, aux moellons et au mortier de chaux jetés pêle-mêle, sans aucun ordre. Ce remplissage permettait aussi de maintenir en place, au moment de la construction, quatre poteaux verticaux de $20 \mathrm{~cm}$ de diamètre en moyenne, plantés et calés dans les angles intérieurs de la partie basse. Ils allaient constituer la structure portante d'un étage entièrement constitué de matériaux périssables.

Tout en gardant à l'édifice un aspect massif et prestigieux, lié à l'utilisation de la pierre, les techniques utilisées dans la construction de cette fortification offraient l'avantage d'être à la fois économiques et rapides à mettre en œuvre. Il s'agit, à l'heure actuelle, du seul exemple de ce type de construction reconnu archéologiquement en Corse mais la documentation écrite peut laisser penser qu'il n'avait rien d'original dans le contexte insulaire. Dans son histoire de la Corse, rédigée durant la seconde moitié du XVe siècle, Pietro Cirneo fait le récit très détaillé de l'attaque d'une fortification à Ghisoni dans le courant du XIIIe siècle (CIRNEO 1834: 174-175). Cette tour, super petram edificata, était naturellement défendue sur trois de ses côtés par des à-pic infranchissables. Pour renforcer le système défensif, un mur fut construit à quatre pas de l'édifice. De même, supra tectum, etsi domus erat mediocri altitudine, fecerat tamen turrim ligneam duorum tabulorum. Ainsi, jugée trop basse, cette domus a été surélevée par la construction d'une tour

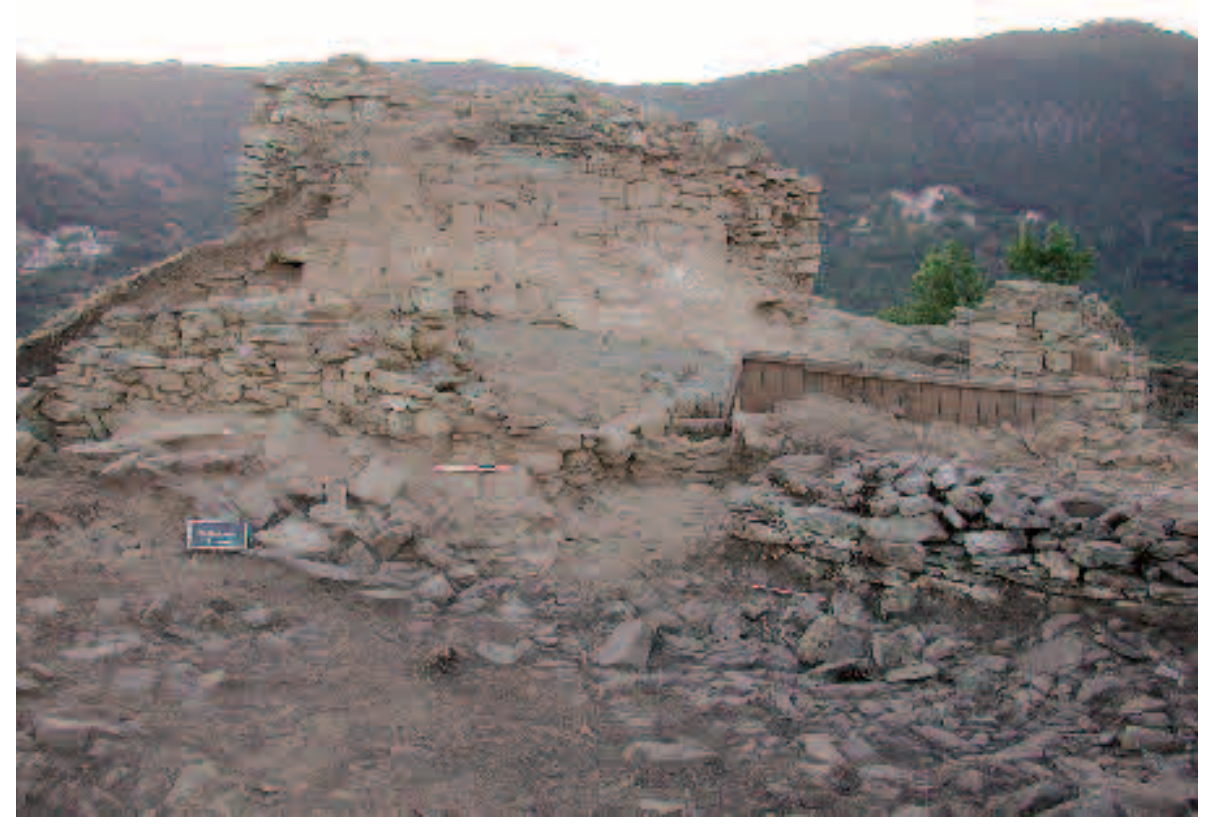

Fig. 6. Castello-di-Rostino, château de Rostino. Vue générale du donjon 2 en cours de fouilles 
en bois de deux niveaux. Bien que le texte de Pietro Cirneo soit de deux siècles postérieurs aux événements, il nous donne l'image exacte et précise d'une réalité matérielle reconnue à Rostino, à la seule différence que, sur ce dernier site, l'élévation en bois faisait partie du projet initial.

L'utilisation simultanée de deux techniques bien différentes dans un édifice pose le problème de l'interférence tradition/innovation. Pourtant, ce n'est pas localement qu'il faudra chercher une solution mais bien, encore une fois, à Pise et dans sa proche région. Les recherches conduites par F. Redi ont montré qu'il existait des maisons et surtout des case-torre construites selon le même mode. La plus intéressante est la tour de Lupeta, un écart situé à quelques kilomètres de Vicopisano. Les restaurations ont permis de découvrir une structure en bois qui reposait primitivement sur une base de pierre. Cette construction de la fin du XIIe siècle fut entièrement englobée dans une maçonnerie à partir du siècle suivant (REDI 1996). A Pise même, les exemples de ce type sont nombreux et l'étude du bâti des XIIe et XIIIe siècles a bien montré que le bois était très souvent associé à la pierre selon des systèmes proches de celui reconnu à Rostino (REDI 1991 et 1996).

Il est donc très vraisemblable que la solution mise en œuvre à Rostino soit élaborée à partir de modèles pisans ${ }^{22}$ bien qu'il existe une tradition locale de la construction en bois. Mais, la méconnaissance du bâti du haut Moyen Age incite à la prudence. Probablement qu'ici, comme dans toutes les régions culturellement proches, le bois a joué un rôle essentiel dans l'architecture des VIIIe-XIe siècles (QUIRÓs CASTILLO 1997). Il n'existe, à l'heure actuelle, aucun exemple connu, mais les découvertes réalisées sur plusieurs sites insulaires témoignent du fait que ce matériau fut d'un usage fréquent durant le bas Moyen Age.

\section{LE BOIS, ENCORE ET TOUJOURS}

Sur le site de Rostino, les traces d'une structure de bois ont été identifiées à quelques mètres en contrebas du donjon. Le plan rectangulaire de ce bâtiment $(3 \times 5 \mathrm{~m}$ environ $) \mathrm{a}$ été restitué grâce à la découverte de trous de poteaux de forme quadrangulaire $(20 \times 15 \mathrm{~cm} ; 20 \times 20 \mathrm{~cm} ; 23 \times 27 \mathrm{~cm})$ entourés de pierres de calage relativement importantes, plantées sur chant pour la plupart. On note aussi la présence de calages de supports verticaux intermédiaires qui se présentent soit sous la forme d'entailles de forme rectangulaire de 20 à $25 \mathrm{~cm}$ de côté, aménagées dans le roc sur 5 à

\footnotetext{
22 Les promoteurs du castrum de Rostino, les marquis Obertenghi, sont étroitement liés, au moins depuis la fin du XIe siècle, à la cité de Pise et possèdent un énorme patrimoine foncier, concédé par l'évêque de Pise, autour de Vicopisano: ISTRIA 2000.
}

$10 \mathrm{~cm}$ de profondeur, soit sous celle de trous circulaires de 10 à $12 \mathrm{~cm}$ de diamètre creusés dans le substrat argileux. Une seule porte a été supposée. Elle devait s'ouvrir dans la paroi nord du bâtiment, face au donjon, et donnait accès à un espace d'environ $10 \mathrm{~m}^{2}$, fermé sur trois côtés par le bâtiment de bois lui-même, par l'enceinte maçonnée qui protégeait le donjon et par un petit mur en pierres sèches, construit sur le rebord du plateau rocheux. Ici, très certainement sous un toit en matériaux périssables, avait été installé un foyer à même le sol. Ce dernier était encombré de fragments de céramique d'importation et de verre, ce qui laisse penser qu'il s'agit d'un habitat d'un certain prestige dont la construction semble remonter aux années $1210-30^{23}$. Le mobilier archéologique provenant du sol le plus récent est, en revanche, peu significatif ${ }^{24}$, mais la destruction de cette structure est toutefois antérieure au début du XIVe siècle, date du réaménagement total de cet espace ${ }^{25}$.

Les structures fouillées dans le sud de la Corse, sur les sites de Corvo et de Capula, pouvaient être assez différentes de celles de Rostino.

Le travail qui a été conduit en 1993 sur la fortification de Corvo (IsTRIA 1995), dans la région de Sartène, a permis de mettre en évidence la présence de structures associées à un château et protégées par un rempart de type cyclopéen, probablement érigé durant la protohistoire mais repris et, par endroits, complété entre le XIIe et le XIVe siècle. Seules deux de ces structures ont été explorées. Leur plan, que l'enlèvement de la couche d'humus, très fine, avait permis de reconnaitre dans les grandes lignes, est très irrégulier et étroitement lié à la morphologie du site et surtout à la présence de blocs rocheux contre lesquels les parois des cabanes prenaient appui. Les murs peuvent être considérés comme des soubassements de pierres d'environ $60 \mathrm{~cm}$ d'épaisseur et $30 \mathrm{~cm}$ de hauteur, formés de deux parements, au centre desquels se trouvaient quelques petits moellons et de la terre. On insistera sur l'absence totale de fondations et d'éboulis. Par conséquent, on peut penser que des parois de bois étaient appuyées et soutenues par ces solins. Les sols intérieurs étaient simplement en terre. Aucune trace des couvertures n'a été rencontrée, ce qui indique qu'elles étaient constituées de matériaux périssables. Le

\footnotetext{
${ }^{23}$ On soulignera principalement la présence d'un fragment de plat ou de bol de protomajolique ligure dans le niveau d'occupation le plus ancien et de tessons de sgraffito archaïques (ISTRIA 2000: 240-241).

${ }^{24}$ Ce mobilier est constitué de fragments de marmites et de testi, c'est-à-dire des plats de fabrication locale destinés à la cuisson des galettes, de quelques tessons d'amphores pisanes ainsi que d'un pied de verre.

25 Cette datation est fournie par des céramiques et une monnaie provenant du niveau supérieur correspondant à la phase de construction de la salle 1.
} 


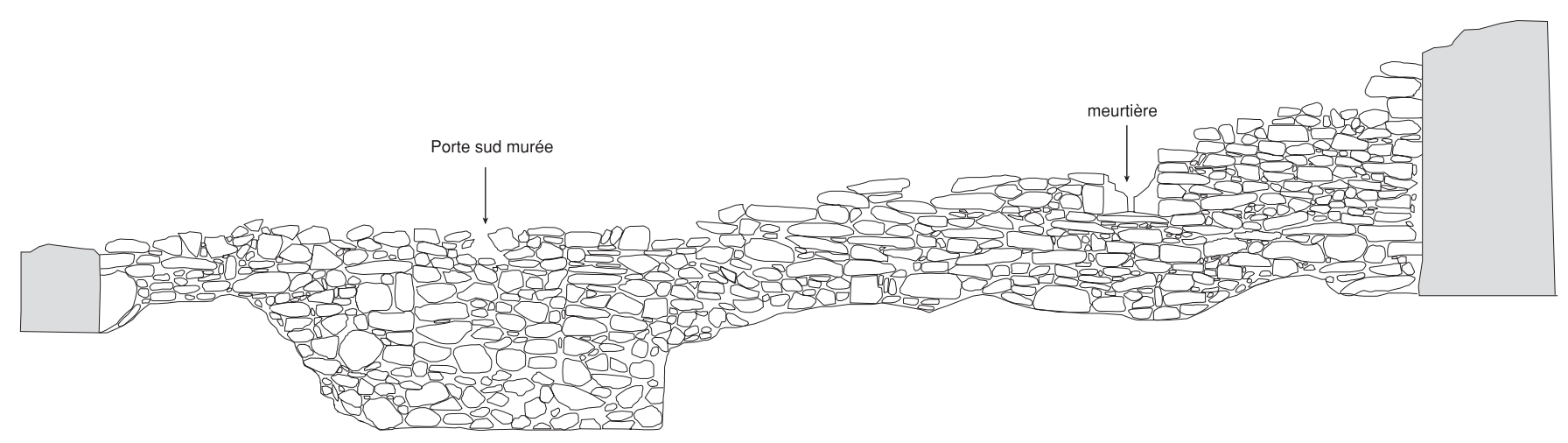

Fig. 7. Castello-di-Rostino, château de Rostino. Elévation du mur sud de la salle 1

mobilier recueilli durant la fouille, autour des foyers, atteste l'utilisation de ces structures comme maisons d'habitation et permet de situer leur abandon vers le milieu du XIVe siècle (ISTRIA 1995).

Une technique de construction identique a également pu être observée par F. de Lanfranchi sur le site de Capula (LANFRANCHI 1978). Elle est documentée non seulement par la présence de petits murets de pierres sèches, par l'absence d'éboulis et de fondations, mais aussi par la présence de trous de poteaux aux angles de la construction. La cabane, située au pied du château, était en partie constituée d'un abri sous roche, fermé par un muret délimitant une surface grossièrement rectangulaire et servant de soubassement aux parois de bois ${ }^{26}$.

Des constructions, très semblables à celles-ci, ont été fouillées par G. Giovanangeli sur le site de l'Ortolo, en Corse-du-Sud, et ont été datées des années 1450-1520 (Giovanangeli 1995). Ainsi, ces quelques exemples témoignent de l'utilisation du bois dans l'architecture rustique durant le tout le bas Moyen Age. Il y à donc une conservation de cette tradition architecturale dans des contextes villageois que l'on ne peut, en aucun cas, considérer comme

\footnotetext{
${ }^{26}$ Le site de Dassargues, dans l'Hérault, a livré une structure rectangulaire, utilisée entre la fin du ve et le début du VIIe siècle - la cabane 2-, dont deux des parois reposaient sur des soubassements de pierres sèches (GARNIER et al. 1995: 36). Mais c'est surtout en Italie qu'il faut rechercher des comparaisons possibles. Ainsi, en Italie septentrionale, les sites de Sant'Antonino de Perti et de Castelseprio ont livré des constructions du même type, datables du très haut Moyen Age (MurIALDo 1996, GELICHI et LiBRENTI 1997). En revanche, à Brescia, en Lombardie, ce sont des structures médiévales du XIIe siècle qui présentent les mêmes caractéristiques (PANAZZA et Brogiolo 1988: 43 à 45 et 60 à 61; Brogiolo 1996: 26 et Fig. 4). L'édifice IV, découvert via Alberto Mario, était constitué très probablement de petits murets en pierres sèches ou liées à la terre, de facture extrêmement grossière. Ils servaient de base à des poutres de bois disposées horizontalement. Ce sont, à l'heure actuelle, les exemples les plus proches, tant chronologiquement, bien sûr, que typologiquement, qui aient été mis au jour en Italie.
}

marginaux même si, au moins depuis la fin du XIIIe siècle, l'architecture de pierre s'est "démocratisée».

\section{APPAUVRISSEMENT DU SAVOIR OU NOUVELLES MODES?}

Dans l'état actuel de nos connaissances, il semble que l'utilisation de la pierre de taille se limite chronologiquement à la fin du XIe et au XIIe siècle ${ }^{27}$. Dès les années 1200 , le réseau d'édifice de culte est déjà bien en place et les équipes de maçons travaillent désormais presque uniquement à la construction de fortifications et de maisons. Contrairement à la Sardaigne où de puissantes familles pisanes et génoises se sont installées et ont érigé des châteaux dans lesquels la pierre de taille est utilisée de manière quasi systématique (Poisson 1990a et b), les promoteurs sont ici des lignages autochtones. Même s'ils entretiennent des contacts réguliers avec la péninsule, cette architecture, surtout de la seconde moitié du XIIIe et du début du XIVe siècle, est marquée par un certain archaïsme, tant dans le plan —rareté des plans réguliers, des tours de flanquement, de fossés...que dans les techniques de construction mises en œuvre. Le moellon informe, issu soit du débitage du substrat géologique présent sur le site même, soit d'un simple ramassage de surface, constitue l'essentiel de la maçonnerie (Fig. 7). Seuls les linteaux, les seuils, voire dans le meilleur des cas, quelques éléments des jambages des portes, font l'objet d'une attention particulière dans l'architecture castrale. L'usage de la gradine, de la broche et du ciseau droit est totalement abandonné au profit du pic. En revanche, le mortier de chaux demeure d'un usage courant. Sa fabrication est même parfaitement maîtrisée. Lors de la construction du donjon I

\footnotetext{
27 En Sardaigne, de nombreux édifices sont encore construits en pierre de taille aux XIIIe et XIVe siècles. Outre les églises d'influence gothique comme la cathédrale d'Iglesias (1284-88) ou celle d'Alghero, on citera, par exemple, les puissantes tours du château de Cagliari et du château Malaspina de Bosa, construites au début du XIVe siècle par Giovanni Capula, qui n'ont pas d'équivalent en Corse.
} 
de Rostino - fin XIIIe-début XIVe siècle-, on a utilisé pour le blocage du mur un mortier de chaux grossier constitué de sable de rivière contenant des éléments de plusieurs millimètres de diamètre. Par contre, les joints recouvrant largement les parements extérieurs, sont constitués d'un enduit fin, très gras et dur, fabriqué avec un sable dont la granulométrie est homogène, sélectionné -les éléments constituants prouvent que sa provenance est différente de celui utilisé pour la construction du mur- et très probablement tamisé. De même, la citerne toute proche et contemporaine du donjon I, a été construite avec exactement le même mortier mais un enduit de tuileau recouvre l'intégralité des parois intérieures. Ici, la chaux a été mélangée à de la terre cuite concassée et du sable très fin et homogène, en quantité réduite. Il revêt ainsi un aspect lisse, une couleur rose et présente l'intérêt d'être solide et parfaitement étanche. Deux couches d'enduits, de nature et d'épaisseur différentes, ont été nécessaires. La première, destinée à régulariser la surface du mur, est constituée d'un mortier de qualité moyenne. Son épaisseur est rarement inférieure à $1,5 \mathrm{~cm}$. La seconde, beaucoup plus fine et homogène, d'environ 0,2 à $0,5 \mathrm{~cm}$ d'épaisseur, forme une surface parfaitement lisse et assure l'étanchéité de la citerne ${ }^{29}$.

La maison villageoise prend, quant à elle, des allures totalement nouvelles. La pierre devient, désormais, un matériau couramment utilisé. Comme dans les châteaux, l'utilisation du moellon brut, issus du débitage de la roche en place ou plus couramment prélevé en surface, est de règle. En revanche, les moellons sont liés à la terre argileuse et non plus au mortier de chaux, y compris sur les sites qui, comme le village de la Mugliunaccia, sont édifiés sur des socles calcaires facilement exploitables (Istria 2000a). L'irrégularité des murs et des plans tout comme l'hétérogénéité générale qui se dégage de ces constructions, laissent penser qu'elles sont l'œuvre de petites équipes d'un faible niveau technologique, voire des villageois eux-mêmes.

\footnotetext{
${ }^{28}$ Ils étaient passés à la truelle dont plusieurs empreintes ont été conservées dans la citerne du château de Lavasina. Il s'agissait ici d'un petit outil de type langue-de-chat; sa longueur a été estimée à environ $9 \mathrm{~cm}$ et sa largeur à $4,5 \mathrm{~cm}$. Dans le donjon du château de San Colombano de Rogliano, on peut observer que le sol a été préparé avec beaucoup de soin avant la pose de ce mortier. Sur le rocher accidenté, les constructeurs ont mis en place une semelle de 50 à $60 \mathrm{~cm}$ d'épaisseur environ, constituée de moellons jetés pêle-mêle et liés par un abondant mortier de chaux. Ce dernier a aussi servi à régulariser le sommet de cette semelle, sur laquelle ont été posées de très larges lauzes bien régulières, de $4 \mathrm{~cm}$ d'épaisseur, recouvertes elles-mêmes par une couche de mortier de $9 \mathrm{~cm}$ d'épaisseur. Le tuileau ne semble présent qu’à la superficie de celle-ci. Il est probable que ces deux mortiers ont été posés immédiatement l'un après l'autre et qu'ils ont fusionné, de telle sorte que la ligne de contact n'est plus visible aujourd'hui.
}

On pourrait admettre, en première analyse, que ces constructions du XIIIe et du début du XIVe siècle sont l'œuvre d'artisans autochtones qui ont hérité d'un savoir importé dans l'île par les maçons du nord de la péninsule Italienne à la fin du XIe et au XIIe siècle, mais qui n'en ont gardé que certains éléments. Ce phénomène pourrait s'expliquer, non pas tant en fonction de leur capacité à assimiler et à transmettre cet héritage technique mais, plus probablement, en fonction de la demande. A partir du XIIIe siècle se mettent en place de grands réseaux de fortifications sous le contrôle d'un personnage ou, plus souvent encore, d'un consortium. Le château perd alors une grande partie de son caractère symbolique, reflet de la puissance et du pouvoir, pour devenir presque essentiellement un instrument de contrôle du territoire et des hommes (IsTRIA 2005). La pierre de taille, plus coûteuse que le moellon, et donc signe de richesse et élément de prestige, disparaît. Quant au bossage, pourtant présent en Sardaigne à la même épo$q^{29} e^{29}$, il n'est jamais utilisé ici. Le donjon lui-même ne devient jamais une structure d'habitat mais reste un pur élément militaire.

La situation apparaît pourtant bien plus complexe et quelques influences continentales se ressentent encore fortement durant la seconde moitié du XIIIe et au XIVe siècle. Sur le site de Cotone, interprété comme un castrum de colonisation génoise, fondé au tout début du XIVe siècle et abandonné une cinquantaine d'années plus tard, l'architecture de pierres sèches rappelle incontestablement les constructions fortifiées contemporaines de Ligurie (IsTRIA, Le château..., ISTRIA 2000a, BENENTE et al. 2000). L'acte de fondation de Castel Lombardo, également un castrum de colonisation génoise, indique de manière très claire que des artisans maçons, tailleurs de pierres et briquetiers étaient envoyés de Ligurie en 1272 pour participer à la construction du village (LOPEZ 1965). D'autre part, même si l'organisation des fortifications corses n'a jamais atteint le degré d'évolution du château sarde de San Michele de Cagliari, où le plan carré est associé à de grosses tours à bossages placées dans les angles, on constate dans trois cas la présence d'un donjon en position centrale par rapport à l'enceinte qui englobe un espace de plan relativement régulier (Serravalle, San Colombano de Rogliano et Avortica), alors que deux modestes tours flanquent l'enceinte d'Avortica. Ces nouvelles dispositions, qui rappellent certaines fortifications de Toscane, de Ligurie ou encore du Frioul, semblent être le résultat de modifications postérieures au milieu du XIIIe siècle et sont, de toute évidence, influencées par les constructions

\footnotetext{
29 Voir par exemple la tour polychrome du palais judical d'Ardara ou encore les tours d'angle du château San Michele de Cagliari.
} 
continentales (IsTRIA 2005). Mais, un autre élément témoigne qu'en matière d'architecture une influence extérieure s'exerce de manière ponctuelle sur la Corse au XIIIe siècle: l'utilisation de la brique.

\section{LA BRIQUE: UN MATÉRIAU RARE POUR UN ÉDIFICE EXCEPTIONNEL}

La brique, qu'elle soit en terre crue ou cuite, apparait comme un matériau de construction de première importance dans le monde méditerranéen antique et médiéval ${ }^{30}$. Pourtant, son utilisation s'est affirmée dans certaines régions plus que dans d'autres. Nous ne retiendrons que les cas de la Toscane et de la Ligurie où les recherches conduites ces vingt dernières années ont montré son rôle fondamental dans l'architecture médiévale ${ }^{31}$. En effet, si le XIIe siècle est considéré dans le nord-ouest de l'Italie comme le siècle de la pierre de taille, le XIIIe est, quant à lui, perçu aujourd'hui comme celui de la brique. Apparu vers 1160 dans les grands centres urbains et principalement à Pise, puis dans le centre de la Toscane en suivant l'axe de la Via Francigena (PARENTI et Quirós CASTILlo 2000) ${ }^{32}$, ce matériau s'est assez rapidement diffusé à travers tout ce secteur nord occidental de la péninsule jusqu'en milieu rural. Cependant, à l'exception des grandes réalisations liées souvent à la fondation de nouveaux bourgs, les «terre nuove» (ABELA 1997), la brique est réservée presque exclusivement, en dehors des villes et avant le XIVe siècle, à l'édification des édifices de culte ou de

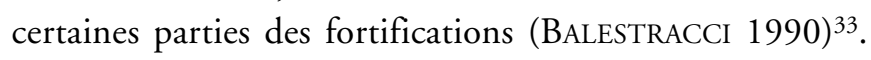

\footnotetext{
${ }^{30}$ Les actes du colloque «La brique antique et médiévale, production et commercialisation d'un matériau", publiés en 2000 dans la Collection de l'Ecole Française de Rome, 272, est, à l'heure actuelle, l'ouvrage le plus récent et le plus complet concernant les problèmes liés à la brique.

31 Nous ne nous intéresserons qu'à ces deux régions qui ont entretenu des rapports politiques et économiques privilégiés avec la Corse durant tout le Moyen Age. Voir par exemple les articles de fonds suivant MANNONI et Milanese 1988; Pittoluga et Quiros Castillo 1998; Parenti et QuiRÓS CASTILLO 2000. Nous renvoyons à l'ouvrage dans lequel est incéré ce dernier article pour une vision plus large du problème de la brique durant l'Antiquité et le Moyen Age et pour les régions où ce matériau a joué un rôle essentiel durant les siècles qui nous intéressent, comme le nord-est de l'Italie, Rome ou le sud-ouest de la France.

32 Au XIIe siècle la brique est principalement utilisée dans la construction des édifices de culte (églises et campaniles) puis dans celle des murs d'enceinte. A Pise, dès la fin du XIIe siècle elle semble aussi utilisée dans les chantiers privés pour l'édification de maisons. En 1182 il est en effet question d'une domus tegularum: REDI 2000.

${ }^{33}$ Le Valdinievole est une situation intéressante qui peut illustrer la diffusion de ce matériau de construction dans les zones périphériques des grands centres urbains, notamment Pise et Lucques. La brique y apparaît dans les chantiers de construction d'église dès 1180 environ (cathédrale de San Miniato et San Salvatore de Fucecchio) alors que ce n'est que durant la seconde moitié du XIIIe siècle qu'on l'utilise de manière plus régulière dans la construction des fortifications situées dans le centre de la vallée, le long de la voie qui unissait les villes de Pistoia et Lucques: Quirós CASTILLO 1997.
}

Pourtant, contrairement à ces régions du nord-ouest de la péninsule Italienne et même à la Sardaigne où sont encore conservés des monuments en élévation ${ }^{34}$, la Corse ne conserve qu'un seul édifice médiéval de ce type. Il s'agit du «palais épiscopal» de Mariana.

Ce bâtiment de taille importante est constitué de deux ailes rectangulaires de dimensions assez comparables $(7 \times 25 \mathrm{~m}$ et $7 \times 27 \mathrm{~m})$ et disposées perpendiculairement l'une par rapport à l'autre de manière à former une cour intérieure dont le côté ouest était fermé par un simple mur de clôture et la partie nord par la cathédrale elle-même. Lespace ainsi enclos est un quadrilatère de $23 \times 25 \mathrm{~m}$ de côtés. Une seule entrée est signalée. Elle est située dans le mur pignon ouest du corps de bâtiment parallèle à la cathédrale. Un mur de refend, percé lui aussi d'une porte, divise cet espace en deux parties très inégales. Ce "palais ${ }^{35}$ " est construit sur les vestiges d'un bâtiment antique ainsi que sur ceux de la basilique paléochrétienne et il est appuyé au mur sud de la cathédrale. Il est donc postérieur à la construction de cette dernière et à celle de la tour, elle-même érigée contre le flanc sud de l'église médiévale. Le plan général de l'ensemble est très proche des quartiers canoniaux du sillon rhodanien et du littoral méditerranéen (ESQUIEU 1992). On y retrouve les bâtiments qui s'organisent autour d'une cour quadrangulaire dans laquelle prend place le clô̂tre qui, toutefois, n’a jamais été formellement reconnu à Mariana.

Les murs en briques, dont l'épaisseur varie de 51 à $55 \mathrm{~cm}$, reposaient sur une semelle de fondation en galets liés à la chaux qui s'apparente à celles des édifices religieux $^{36}$. La technique de construction mise en œuvre est toutefois très particulière. Les galets, de calibre homogène, ont été superposés avec soin et en assises horizontales régulières, parfois en arête de poisson. Les dernières assises sont constituées de moellons régularisés, parfois équarris ${ }^{37}$, de manière à former une base parfaitement horizontale pour la

\footnotetext{
34 Pour la Sardaigne, qui s'impose comme un point de comparaison inévitable en raison de la proximité géographique des deux îles $(10 \mathrm{~km}$ environ) et des relations qu'elles ont entretenu avec Pise et Gênes, il n'existe pas, a notre connaissance d'étude consacrée spécifiquement aux édifices en briques. Nous renvoyons donc à l'ouvrage de R. Coroneo (1993) qui présentent une intéressante série de monuments religieux de ce type. Voir en particulier l'église S. Nicola di Quirra a Villaputzu, entièrement construite en briques vers la fin XIIe-début XIIIe siècle.

${ }^{35}$ Le terme est utilisé pour la première fois par G. Moracchini-Mazel, auteur des fouilles qui, dans les années 1960, permirent de dégager ces vestiges.

36 C'est une fondation identique que l'on peut apercevoir dans la cavité qui a été aménagée après les fouilles des années 1950-60 dans l'abside de la cathédrale médiévale (début XIIe siècle) de Mariana.

37 Beaucoup de ces moellons semblent avoir été récupérés sur le chantier de la cathédrale.
} 
pose des briques. Approximativement verticale, cette semelle a une hauteur minimum ${ }^{38}$ de $1,40 \mathrm{~m}$ et déborde d'une quinzaine de centimètres par rapport à l'épaisseur du mur sur chacune des faces. Sa mise en place a été imposée ici par l'absence de substrat rocheux. La construction est installée sur des alluvions fluviatiles très récentes qui recouvrent un substrat constitué de galets. Un sous-sol par conséquent instable qui, sans cela, n'aurait pu supporter le poids d'une construction d'une telle ampleur, même si ses murs étaient en briques. Malgré leur massivité apparente, ses fondations sont relativement fragiles en raison de l'utilisation de galets dont la forme, proche de la sphère, canalise mal les forces verticales exercées par le poids de la construction. Ainsi, en certains endroits, les assises présentent des marques d'affaissement qui, toutefois, n'ont sans doute pas mis en péril la stabilité du mur qui les surmontaient ${ }^{39}$.

Sur ces fondations s'élèvent encore aujourd'hui deux à quatre assises de briques de réemplois et d'autres dont les dimensions $(29,9$ à $30,8 \times 12$ à $12,6 \times 5,5$ à $6,5 \mathrm{~cm})$ sont complètement différentes de celles utilisées pour la construction des autres structures visibles actuellement sur le site (Fig. 8$)^{40}$. Nous sommes donc certainement en présence de matériaux confectionnés au Moyen Age, spécifiquement pour l'édification de ce grand bâtiment qui comportait peut-être un étage. Nous ne savons bien entendu rien sur l'endroit où ces briques ont été produites mais la présence d'importants filons d'argile dans la plaine de Mariana, notamment au nord du site où fut installée une briqueterie à l'époque moderne, laisse penser à une fabrication quasiment in situ par des artisans itinérants (PARENTI et QUIRÓs CASTILLO 2000). On peut exclure, pour l'heure, la possibilité d'une importation de ces briques dans la mesure où, aussi bien les caractéristiques de la pâte que les dimensions ne correspondent pas à celles produites dans les régions d'où elles auraient pu provenir, c'est-à-dire la Ligurie et la Toscane.

En ce qui concerne la datation de cet édifice, nous avons vu qu'il est indiscutablement postérieur à la cathédrale (1119) et à la tour. Le site est abandonné définitivement durant le bas Moyen Age comme l'atteste la documentation

38 La base de cette semelle de fondation n'est pas visible actuellement. Il est fort probable qu'en raison de la texture du sol, très meuble, et de l'importance des bâtiments projetés, ces fondations s'appuient sur des constructions antiques sous-jacentes. C'est le cas dans la partie orientale où les murs antérieurs (paléochrétiens) ont été conservés sur une élévation relativement importante.

39 Nous manquons totalement d'informations sur l'utilisation de cet édifice. Les fouilles conduites par G. Morachini-Mazel n'ont laissé aucune documentation.

40 Seuls deux caveaux fouillés anciennement par G. Moracchini Mazel étaient construits avec ces mêmes briques. Ils ne sont plus visibles aujourd'hui: MORACCHINI-MAZEL 1967: 12.

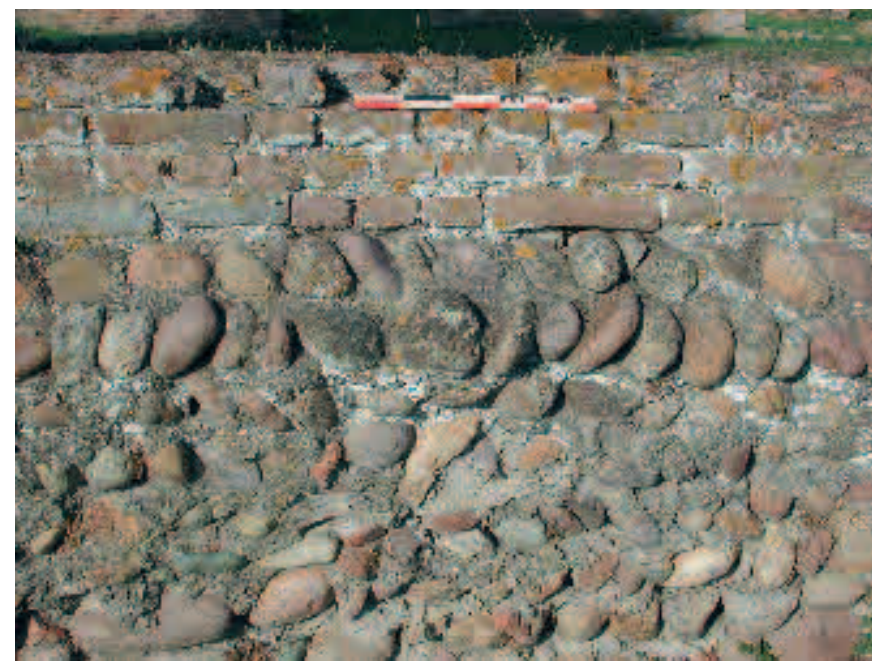

Fig. 8. Lucciana, site de Mariana, détail du mur sud du «palais épiscopal»

écrite qui signale l'installation de la résidence épiscopale à Cortecatto puis à Belfiorito (village actuel de Vescovato) vers le milieu du XIIIe siècle ${ }^{41}$, mais aussi par les fouilles archéologiques qui n'ont livré que peu de céramiques des XIVe et XVe siècles en regard des productions antérieures. Un manuscrit de la bibliothèque vaticane, daté du XVIe siècle,

41 Dans le manuscrit Urb. Lat. 969 de la bibliothèque vaticane, on peut lire un passage relatif au village de Vescovato peut-être écrit, ou du moins très proche du récit de Marc'Antonio Ceccaldi (1521-1561) —d'ailleurs originaire de Vescovato: «La villa del Vescovato, non sono piu de 200 anni che fú habitata in la seguente forma» le manuscrit des chroniques de Giovanni della Grossa, publié par l'abbé Letteron en 1888 dans le Bulletin de la Société des sciences historiques et naturelles de la Corse, indique: «il n’y à pas plus de 300 ans que Vescovato est habité» (p. 162)—, essendo destrutta la città de Mariana, il vescovo de quella si ritiro ad'habitare in uno luoco longi dal Vescovato circa un' miglio dove dal suo nome chiamo quel luoco Cortecatto et cosi duro questa habitatione molti anni con la residenza vescovale. Come luoco piu atto à ridur le reliquie della città destrutta» (for $16 r$.$) . Le texte raconte par la suite que l'évêque déplaça un peu plus$ tard sa résidence à Vescovato (Belfiorito). Le castrum de Belfiorito est mentionné pour la première fois en 1289 (Archivio di Stato de Gênes, Archivio segreto, n. ${ }^{\circ}$ 2725/62, 15 août 1289). Selon Giovanni della Grossa c'est l'évêque Opizo Pernice Cortinco qui s'installe le premier à Belfiorito. Un Opizo Pernice Cortinco de Lumito est mentionné dans le document de 1242. Il est alors procurateur de l'abbaye de Montecristo. Pier'Antonio Monteggiani, qui écrivait à la fin du XVe siècle, évoque, en 1477 , une «veduta générale à l'église de la Canonica, où s'élevait autrefois la ville de Mariana...». Vers 1530, Mgr Agostino Gustiniano fait de l'église cathédrale la description suivante: «Cette église est si mal entretenue et si peu soignée qu'on peut l'appeler une étable d'animaux, plutôt qu'un temple consacré au culte divin. Et certes, les diocésains de Mariana ont bien le droit de se plaindre qu'un monument si beau et si digne de respect soit ainsi délaissé; au moins si on y entretenait deux ermites, ceux-ci pourraient préserver cette belle église des profanations des boeufs, des vaches et des porcs. Mais peut-être faut-il attribuer l'état de délabrement dans lequel se trouve cet édifice aux malheurs des temps autant qu'à la négligence des prélats...». Une restauration de la cathédrale fut envisagée par Mgr de Fornari entre 1463 et 1469 mais jamais réalisée (MORACCHINI-MAZEL 1967: 218). 
indique que la ville de Mariana a, par ailleurs, été détruite au XIIIe siècle ${ }^{42}$. Une attribution de cette construction à la fin du XIIe ou au XIIIe siècle semble en accord avec le type d'architecture qui se développe dans le nord-ouest de la péninsule Italienne à partir de la seconde moitié du XIIe siècle.

Le palais de Mariana est donc le seul édifice médiéval corse connu qui fut construit en briques. Bien qu'implanté dans ce qui fut le centre religieux et peut-être même politique, ou du moins symbolique, le plus important de l'île ${ }^{43}$, il ne servit pas de modèle et ce n'est qu'au XVe siècle que l'usage de la brique va se répandre dans l'île et plus particulièrement dans les forteresses côtières contrôlées par Gênes.

\section{L'ENCLAVE GÉNOISE DE BONIFACIO: UNE EXCEPTION DANS LE CADRE INSULAIRE}

La colonie génoise de Bonifacio a été fondée en 1195 sur une presqu'île de l'extrémité sud de la Corse. Peuplée principalement de ligures, elle s'est développée très rapidement grâce à son activité commerciale internationale (HEERs 1979). Aux XIIIe et XIVe siècles, elle est encore le seul centre urbain de l'île. Le castrum s'organise, dès le départ, selon un plan orthonormé irrégulier construit autour de quatre axes orientés Est-Ouest et au centre duquel se trouve l'église paroissiale Sainte-Marie-Majeure datant, très probablement, de la fin du XIIe siècle (GAYRAUD 1976). Au moins quatre autres églises médiévales sont conservées sur le plateau mais nous ne disposons de données chronologiques sûres que pour l'église conventuelle de Saint-Dominique, érigée entre 1270 et 1343 (Pergola et Istria 2001, vol. II: 34 et 42) et fortement inspirée par l'église San Matteo de Gênes. Si l'influence gothique se devine, particulièrement dans l'organisation de la façade occidentale constituée d'une porte inscrite dans une arcature en tiers-point à multiples ressauts et surmontée d'une grande rosace, cet édifice reste très sobre et de petites dimensions, mal éclairé par d'étroites fenêtres

\footnotetext{
42 Voir note précédente. Même si la date donnée par ces textes — qui seraient donc postérieurs à cet événement de deux à trois siècles - incite à la plus grande prudence, on peut admettre sans aucune condition que Mariana était bien détruite avant le milieu du XVIe siècle puisque Marc'Antonio Ceccaldi, né à Vescovato même en 1521, meurt en janvier 1561 et arrête son récit en 1559.

43 Seule cathédrale consacrée, qui plus est par l'archevêque de Pise et le cardinal de S. Susanna de Rome qui portait alors le titre de légat pontifical. Cette grande cérémonie durant laquelle tous les évêques de l'île jurèrent fidélité à l'archevêque est un moment clé dans l'histoire de la réforme de l'Eglise de Corse mais aussi dans celle de la domination de l'île par les puissances du nord-ouest de la péninsule. Le choix de Mariana semble être lié à l'importance de la ville durant l'Antiquité tardive et bien sûr de son évêché y compris durant le haut Moyen Age, documenté non seulement par les multiples restaurations de la cathédrale et du baptistère —qui reçoit un décor sculpté probablement vers le viIIe siècle- mais aussi par le mobilier archéologique: (Pergola et Di ReNZO 2001).
}

meurtrières qui rappellent celles des constructions romanes. Malgré l'intégration de fines colonnettes aux angles des piles, les arcatures basses et étroites qui séparent les trois vaisseaux conferent à cet espace intérieur un aspect lourd et massif, à peine compensé par la blancheur des murs.

D'autre part, 14 maisons attribuables aux XIIIe et XIVe siècles ont été recensées à l'intérieur des murs de la cité. Elles présentent toutes des caractéristiques classiques de l'architecture civile génoise et plus particulièrement de la maison dite «mercantile». Le regroupement des fonctions d'habitation et commerciales dans une seule unité induit une organisation spécifique de chaque niveau. Le rez-dechaussée, s'ouvrant sur la rue par de grandes arcades légèrement brisées et à double ressauts, abrite les boutiques alors que les étages accueillent les espaces privés. La façade principale est décorée de frises et de bandes arcaturées reposant sur de petits modillons moulurés mais aussi de fenêtres géminées ou de baies triples avec colonnettes (Grossi BiANCHI et POLeGgi 1980; Ferrando Cabona 1996; Toma 1999).

L'ensemble édifices de culte et maisons présente un caractère très homogène. Les blocs de calcaire prélevés dans des secteurs très proche de la ville sont utilisés de manière systématique pour la construction des façades donnant sur la rue alors que les murs latéraux et arrières sont souvent constitués de moellons liés à la chaux. Il y a donc, dans le cas de Bonifacio, une continuité technique ininterrompue du XIIe au XIVe siècle au moins, lié au statut même de la ville et surtout à ses contacts permanents avec Gênes. Par conséquent, plus qu'une permanence des traditions insulaires romanes, il faut voir ici l'importation par les colons de modèles propres à Gênes et incontestablement le recours systématique à des artisans continentaux qui n'ont pas diffusé leur savoir en dehors du territoire du castrum $^{44}$.

\section{CONCLUSION}

La Corse médiévale est une région marginale où peu de réalisations majeures voient le jour. Elle n'en demeure pas moins d'un intérêt certain pour l'étude des échanges et des transferts de savoir-faire dans le bassin nord-occidental de la Méditerranée. Même si notre connaissance du haut Moyen Age est encore bien trop limitée, pour ne pas dire nulle, il apparait que l'affirmation du pouvoir pontifical par l'intermédiaire de l'évêque de Pise est un moment clé de l'histoire de l'île. La construction d'un réseau d'édifices de culte permet l'introduction, puis la diffusion, de procédés techniques remis au goût du jour sur les grands chantiers

${ }^{44}$ Les statuts du XIIIe siècle de la ville de Bonifacio font clairement allusion à la présence, parmi les sergents, de huit maîtres-maçons, cinq tailleurs de pierre et 25 manoeuvres. 
continentaux peu de temps auparavant, à San Pietro a Grado et à la cathédrale de Pise tout particulièrement. C'est alors une véritable renaissance de l'architecture que connaît la Corse entre le dernier tiers du XIe et la fin du XIIe ou au plus tard le tout début du XIIIe siècle.

L'introduction de ces savoir-faire, et particulièrement de la pierre de taille et du mortier de chaux, accompagne la diffusion du style roman et de modèles proprement toscans. Au-delà de l'architecture religieuse, la tour de Rostino, associant base de pierres et étage(s) de bois, et celle de Mariana, en sont les meilleurs exemples. Ce processus se fait par le déplacement d'équipes spécialisées dont il est possible de suivre l'itinéraire depuis la Toscane jusqu'en Corse en passant, bien souvent, par la Sardaigne.

L'acquisition de ce savoir par des équipes locales reste un problème ouvert pour le XIIe siècle. En revanche, au XIIIe siècle, ce sont probablement elles qui travaillent à la construction des forteresses. Mais, ces maçons ne semblent avoir retenu que certains éléments de ce savoir dont, principalement, la fabrication du mortier de chaux et de tuileau. Pourtant, encore au XIIIe et au début du XIVe siècle, ils ne travaillent pas dans un monde clôt. Les influences extérieures se font encore sentir dans l'évolution des plans de quelques châteaux par exemple, mais aussi dans la transformation de l'outillage. Broche, gradine et ciseau droit sont abandonnés au profit du pic, mieux adapté au type d'appareillage mis en œuvre. Même si dans l'architecture rustique, c'est-à-dire dans la maison du castrum, les points communs avec la Toscane ou la Ligurie orientale existent, peut-être en raison de l'arrivée d'artisans chargés de construire les castra de colonisation génois ou pisans, il existe désormais une identité insulaire qui s'inscrit dans un contexte culturel englobant au moins le nord de la Toscane et la Lunigiana. La situation de Bonifacio, seule ville de l'île, est toute différente et s'oppose fondamentalement à celle du monde rural. Ici, l'influence de Gênes est prépondérante et la présence, aux XIIIe et XIVe siècles, de maçons ayant travaillés dans la cité ligure est une quasi-certitude.

En somme, entre le XIe et le XIVe siècle, l'évolution des techniques de construction dans l'île est étroitement liée à la conjoncture historique et donc aux relations qui la liaient aux deux puissances étrangères, Pise et Gênes. Pourtant, peut-on véritablement parler d'un transfert de savoirfaire? Il faudrait pour cela que les artisans insulaires aient assimilé ces techniques pour les mettre en ouvre à leur tour. Létude architecturale de castrum de Rocca San Silvestro, en Toscane, a bien montré que les habitants du village ont perpétué des manières de faire importées par des équipes spécialisées (BIANCHI 1995). En Corse, ce legs n’est que partiel. Plus exactement, les maçons n'en ont retenu que certains aspects, répondant ainsi à un besoin et à une demande: la pierre de taille n'est qu'un artifice esthétique, symbole de prestige et de puissance, mais ne peut garantir à elle seule la solidité de l'architecture. Le contexte politique et économique des XIIIe-XIVe siècles, marqués par l'arrêt de la construction d'édifices de culte, par la transformation du statut du castrum ainsi que par le contrôle du commerce, a donc forcément conditionné les choix des maîtres d'œuvre et, au-delà, l'évolution de l'architecture et des techniques de construction.

\section{Bibliographie}

ABELA E., 1997, L'uso del laterizio nella fondazione delle "terre nuove»: il caso di piazza Bertoncini a Castelfranco di Sotto (Pisa), in I congresso Nazionale di Archeologia Medievale, 29-31 mai 1997, Pise, Firenze, pp. 77-79.

BALESTRACCI D., 1990, I materiali da costruzione nel castello medievale, in R. Francovich, M. Milanese (dir.), Lo scavo archeologico di Montarrenti e i problemi dell'incastellamento medievale. Esperienze a confronto, "Quaderni del dipartimento di archeologia e storia delle arti sezione archeologia - Universita di Siena», Firenze, pp. 227-242.

BAUD A., et al., 1996, L'échafaudage dans le chantier médiéval, Documents d'Archéologie en Rhône-Alpes, 13.

BENENTE et al. 2000, Gli scavi del "castrum Rapallinum» (Monte Caste$10^{\circ}$ e del "castrum Lasaniae» (Monte Pegge), in II congresso Nazionale di Archeologia Medievale, 28 settembre-1 ottobre 2000, Brescia, Firenze, pp. 161-169.

BiANCHI G., 1995, L'analisi dell'evoluzione di un sapere tecnico per una rinnovata interpretazione dell'assetto abitativo e delle strutture edilizie del villaggio fortificato di Rocca S. Silvestro, in BOLdrini E., FrancoVICH R. (dir.), Acculturazione e mutementi, prospettive nell'archeologia medievale del Mediterraneo, Florence, pp. 361-396.

BiANCHI G., 1996, Trasmissione dei saperi tecnici e analisi dei procedimenti costruttivi, Archeologia dell'architettura, I, pp. 53-64.

Brogiolo G.P., 1996, Urbanistica ed edilizia nei quartieri orientali di Brescia nel XII secolo, in De Menicis E., Guidoni E., 1996, Case e torri medievali, Atti del II. o convegno di studi, La città e le case. Tessuti urbani, domus e case-torri nell'Italia Comunale (secc. XI-XV), Città della Pieve, 11-12 dicembre 1992, Roma, pp. 22-27.

Cirneo P., 1834, Petri Cyrnaei, de Rebus Corsicis, éd. Grégori C., Paris.

Coroneo R., 1993, Architettura romanica dalla metà del mille al primo'300, Banco di Sardegna, Nuoro.

Coroneo R., 2004, Il «romanico d'importazione» in Sardegna e in Corsica: crisi e validità di un modello storiografico, in QuiNTAVALLE A.C. (dir.), 2004, Medioevo: arte lombarda. Atti del Convegno internazionale di studi, Parma, 26-29 settembre 2001, Milan, p. 440-456.

Duval N., 1995, Les premiers monuments chrétiens de la France, 1, Sud-Est et Corse, Paris.

EsQUIEU Y., 1992, Autour de nos cathédrales. Quartiers canoniaux du sillon rhodanien et du littoral méditerranéen, Monographie du CRA, 8, CNRS Editions, Paris.

ESQUIEU Y., 1997, L'archéologie du bâti en France, Archeologia dell'architettura, II, pp. 133-140.

Fabiani P., MennuCCI A., NenCI C., 1997, Indagini sui paramenti murari esterni del duomo di Pisa: rapporto preliminare, I congresso nazionale di archeologia medievale, 29-31 mai 1997, Pise, Florence, pp. 449-455.

FERrANDo Cabona I., 1996, Case genovesi e case dei liguri fra Medioevo ed età moderna: prime considerazioni da analisi archeologiche, in DE MINICIS E. et GUIDONI E. (dir.), Case e torri medievali, I, Rome, pp. 41-48.

Gallo N., 1995, Caratteri architetonici e strutturali delle torri medievali nell'antica diocesi di Luni, Castellum, 37, pp. 47-60.

Gallo N. et Marselli G., 1989, Caratteri e aspetti tecnici di alcuni impianti fortificati in area lunense tra altomedioevo e medioevo, Giornale Storico della Lunigiana, XI, pp. 109-122. 
GAYRAUD R. P., 1976, Les fouilles de Bonifacio: reflexions sur l'urbanisme médiéval, Archeologia Corsa, I, pp. 276-294.

GeLICHI S., LibRENTI M., 1997, L'edilizia in legno altomedievale nell'Italia del nord: alcune osservazioni, in I congresso nazionale di archeologia medievale, 29-31 maggio 1997, Pisa, Florence, pp. 215-220.

GiovanANGeli G., 1995, Le village de l'Ortolo: archéologie et histoire d'un village déserté, in Patrimoine d'une île. Recherches récentes d'archéologie médiévale en Corse, Ajaccio, pp. 23-27.

Grossi Bianchi L., POlEgGi E., 1980, Una città portuale del medioevo, genova nei secoli X-XVI, Gênes.

HEERS J., 1979, Un exemple de colonisation médiévale: Bonifacio au XIII siècle, in Société et économie à Gênes (XIVe-XVe siècles), Londres.

IstRIA D., 1995, Les castelli de Cotone et de Corvo: premiers résultats archéologiques, in Patrimoine d'une île. Recherches récentes d'archéologie médiévale en Corse, Ajaccio, pp. 29-34.

IsTRIA D., 2000, Les marquis Obertenghi dans le nord de la Corse (fin du XIe-milieu du XIVe siècle). La mise en place d'un réseau de fortifications, Mélanges de l'Ecole française de Rome, Moyen Age, 112-1, pp. 225-258.

ISTRIA D., 2000a, Les maisons de la première moitié du XIVe siècle dans le castrum du nord de la Corse, Bulletin Monumental, 158-IV, pp. 305-322.

IsTRIA D., Le château..., 2002, Le château, l'église et l'habitat dans le nord de la Corse aux XIIe et XIIIe siècles, Mélanges de l'Ecole française de Rome, Moyen Age, 114-1, (à paraître).

IstRia D., Di RenZo F., 2001, Le paysage chrétien de la Corse médiévale, in Corsica christiana, 2000 ans de christianisme, Catalogue de l'exposition du Musée de Corte, Ajaccio, pp. 126-140.

IsTRIA D., 2005, Pouvoirs et fortifications dans le nord de la Corse, XIe-XIVe siècles, Ajaccio.

LANFRANCHI F. de, 1978, Capula: quatre millénaires de survivances et de traditions. Levie.

LOPEZ R.S., 1965, Da mercanti a agricoltori: aspetti della colonizzazione genovese in Corsica., in Homenaje a Jaime Vicens Vives, Universidad de Barcelona, Facultad de filosofia y letras, I, Barcelona, pp. 525-532.

Mannoni T., Milanese M., 1988, Mensiocronologia, Archeologia e restauro dei monumenti, Firenze, 1988, pp. 383-402.

Moracchini-Mazel G., 1967, Les églises romanes de Corse, Paris.

MoraCCHINI-MAZEL G., (éd.) 1984, La féodalité corse et ses relations avec les cités maritimes. Actes de la table ronde de Biguglia, "Cahiers Corsica", 106-110.

MurAtori L.A., 1725, Rerum Italicarum Scriptores, Milan.

Murialdi G., 1996, Perti: un territorio rurale del Finale tra la Preistoria e l'Eta Moderna, Istituto internazionale di Studi Liguri, Finale Ligure.

Panazza G., Brogiolo G.P., 1988, Ricerche su Brescia altomedioevale, vol. 1. Ateneo di Brescia, Accademia di scienze lettere ed arti.

Parenti R., Fabiani P., Mennucci A., 1997, Linee di progetto per la conoscenza delle strutture meteriali del duomo di Pisa, Archeologia dell'architettura, II, pp. 47-52.

Parenti R., Quirós Castillo J.-A., 2000, La produzione dei mattoni della Toscana medievale (XII-XVI s.): un tentativo di sintesi, in La brique antique et médiévale, production et commercialisation d'un matériau, "Collection de l'Ecole Française de Rome», 272, pp. 219-235.
Pergola Ph., 1979, Une pieve rurale corse: Santa Maria di Talcini; problèmes d'archéologie et de topographie médiévales insulaires, Mélanges de l'Ecole Française de Rome, Moyen Age, 91, pp. 89-111.

Pergola Ph., 1980a, Lo scavo della pieve di Cinarca in Corsica; nuove prospettive per l'archeologia medievale insulare, Archeologia Medievale, VII, pp. 467-474.

Pergola Ph., 1980b, San Ghjuvan Battista pieve de Cinarca à Sari d'Urcinu, Archeologia Corsa, IV, pp. 103-112.

Pergola Ph., 1999, La Corse, in Alle origini della parrocchia rurale (IV-VIII sec.). Atti della giornata tematica dei Seminari di archeologia cristiana, 19 mars 1998, Cité du Vatican, Sussidi allo studio delle antichità cristiane, 12, pp. 205-213.

Pergola Ph., Istria D., 2001, Corsica christiana, 2000 ans de christianisme, Catalogue de l'exposition du Musée de Corte, Ajaccio.

Pergola Ph., Di Renzo F., 2001, Cités et campagnes de Corse de la fin de l'Antiquitié et du haut Moyen Age, in Corsica christiana, 2000 ans de christianisme, Catalogue de l'exposition du Musée de Corte, Ajaccio, pp. 106-124.

PitToluga D., Quirós CASTILlo J.-A., 1998, Mensiocronologia dei laterizi della Liguria e della Toscana: due esperienze a confronto, in $I$ congresso nazionale di archeologia medievale, 29-31 mai 1997, Pise, Firenze, pp. 460-463.

PoIsSON J.-M., 1990a, L'érection des châteaux dans la Sardaigne pisane (XIIIe s.) et ses conséquences sur la réorganisation du réseau d'habitats, Château Gaillard, XIV, pp. 352-366.

POISSON J.-M., 1990b, Castelli medievali di Sardegna: dati storici e dati archeologici, in Francovich R., MilaneSe M. (dir.), Lo scavo archeologico di Montarrenti e i problemi dell'incastellamento medievale. Esperienze a confronto, "Quaderni del dipartimento di archeologia e storia delle arti sezione archeologia - Università di Siena», Florence, pp. 191-204.

QUIRÓS CASTILLO J.-A., 1997, Interpretacion historica y arqueologica de las trasformaciones de las tecnicas constructivas medievales de la Valdinievole (Toscana), Archeologia dell'Archiettura, II, pp. 113-120.

RAT P., 1996, Les pierres de l'abbatiale de Tournus, la géologie et l'homme, Centre international d'études romanes, XCV, pp. 169-204.

REDI F., 1984, Cascina, edilizia medievale e organizzazione del territorio, I, Pise.

REDI F., 1991, Pisa com'era: archeologia, urbanistica e strutture materiali (secoli V-XIV), Naples, 1991.

REDI F., 1996, Reperti archeologici dell'edilizia medievale pisana a confronto con la documentazione archivista coeva, in DE MINICIS E. et Guidoni E. (dir.), Case e torri medievali, I, Rome, pp. 89-100.

REDI F., 1997, Vicopisano e il suo territorio: un'esperienza di archeologia globale, in I congresso nazionale di archeologia medievale, 29-31 mai 1997, Pise, Florence, pp. 147-151.

REDI F., 2000, I laterizi nell' edilizia medievale a Pisa e a Lucca, dans $\mathrm{La}$ brique antique et médiévale, production et commercialisation d'un matériau, "Collection de l'Ecole Française de Rome», 272, pp. 193-218.

Redi, F. et Fanucci Lovitch M., 1998, Nuovi studi di storia e di archeologia su Vicopisano, Bollettino storico pisano, 45.

Toma M., 1999, Una casa mercantile a Genova tra medioevo e seicento, Archeologia dell'architettura, IV, pp. 195-209. 